\title{
Model Predictive Control for Indoor Thermal Comfort and Energy
}

\section{Optimization Using Occupant Feedback}

\author{
Xiao Chen", Qian Wang ${ }^{\mathrm{a},}$, and Jelena Srebric ${ }^{\mathrm{b}}$ \\ ${ }^{a}$ Department of Mechanical and Nuclear Engineering, The Pennsylvania State University, \\ University Park, PA 16802 \\ ${ }^{b}$ Department of Mechanical Engineering, University of Maryland, College Park, MD 20742
}

\begin{abstract}
This study developed two model predictive control (MPC) algorithms, a certainty-equivalence MPC and a chance-constrained MPC, for indoor thermal control to minimize energy consumption while maintaining occupant thermal comfort. It is assumed that occupant perceptions of thermal sensation can be continually collected and fed back to calibrate a dynamic thermal sensation model and to update the MPC. The performance of the proposed MPCs based on Actual Mean Vote (AMV) was compared to an MPC using Fanger's Predicted Mean Vote (PMV) as the thermal comfort index. Simulation results demonstrated that when the PMV gives an accurate prediction of occupants' AMV, the proposed MPCs achieve a comparable level of energy consumption and thermal comfort, while it reduces the demand on continually sensing environmental and occupant parameters used by the PMV model. Simulation results also showed that when there is a discrepancy between the PMV and AMV, the proposed MPC controllers based on AMV expect to outperform the PMV based MPC by providing a better outcome in indoor thermal comfort and energy consumption. In addition, the proposed chance-constrained MPC offers an opportunity to adjust the probability of satisfying the thermal comfort constraint to achieve a balance between energy consumption and thermal comfort.
\end{abstract}

\section{Keywords}

Model Predictive Control (MPC), Dynamic Thermal Sensation (DTS), data-driven model, energy consumption, Actual Mean Vote (AMV), Extended Kalman Filter (EKF)

\footnotetext{
* Corresponding author. The Pennsylvania State University, 325 Leonhard Building, University Park, PA 16802. Tel:(814)8658281; Fax:(814)8659693; Email:quw6@psu.edu
} 


\begin{tabular}{|c|c|c|c|}
\hline \multicolumn{4}{|c|}{ Nomenclature } \\
\hline$A M V$ & Virtual occupants' actual mean vote & $q$ & Slack variable for constraint \\
\hline$A_{w}$ & Wall area, $\mathrm{m}^{2}$ & $q_{\text {vent_flow }}$ & Ventilation heat flow, W \\
\hline$\alpha$ & Percentage of violation in chance constraint & $R_{i j}$ & $\begin{array}{l}\text { Thermal resistance between node } i \text { and } j \text {, } \\
\text { K/W }\end{array}$ \\
\hline$C_{i}$ & $\begin{array}{l}\text { Thermal capacitance of ith node in thermal } \\
\text { network model, J/K-s }\end{array}$ & $R H$ & Relative humidity \\
\hline$C L O$ & Occupants' clothing insulation & $T_{a}$ & Ambient air temperature, $\mathrm{K}$ \\
\hline$c_{p a}$ & Specific heat of air, $\mathrm{J} / \mathrm{Kg}-\mathrm{K}$ & $T_{i}$ & $\begin{array}{l}\text { Temperature of } i t h \text { node in thermal } \\
\text { network model, } \mathrm{K}\end{array}$ \\
\hline$c_{p w}$ & Specific heat of water vapor, $\mathrm{J} / \mathrm{Kg}-\mathrm{K}$ & $T_{o}$ & Outdoor air temperature, $\mathrm{K}$ \\
\hline$d$ & Offset parameter & $T_{m r}$ & Mean radiant temperature, $\mathrm{K}$ \\
\hline$e$ & Process noise & $T_{N}$ & Adjacent room temperature, $\mathrm{K}$ \\
\hline$G_{\text {int }}$ & Internal gain, $\mathrm{W}$ & $T S$ & $\begin{array}{l}\text { Predicted thermal sensation in a generic } \\
\text { model }\end{array}$ \\
\hline$h$ & Convection coefficient, $\mathrm{W} / \mathrm{m}^{2}-\mathrm{K}$ & $\Delta T$ & Sampling time, s \\
\hline$h_{i n}$ & Outdoor specific enthalpy of moist air, $\mathrm{J} / \mathrm{Kg}$ & $t$ & Time step \\
\hline$h_{\text {out }}$ & Indoor specific enthalpy of moist air, $\mathrm{J} / \mathrm{Kg}$ & $u$ & Control input, $\mathrm{W}$ \\
\hline$h_{w e}$ & Evaporation heat of water at 0 Celsius, $\mathrm{J} / \mathrm{Kg}$ & $V_{a}$ & Air velocity, $\mathrm{m} / \mathrm{s}$ \\
\hline$J_{t}$ & Cost at time step $t$ in MPC formulation & $v$ & Measurement noise \\
\hline$k$ & Conduction coefficient, W/m-K & $W_{\text {in }}$ & Indoor humidity ratio \\
\hline$K_{p}$ & Proportional gain & $W_{\text {out }}$ & Outdoor humidity ratio \\
\hline$K_{I}$ & Integral gain & $W_{s}$ & Saturation humidity ratio \\
\hline$l$ & Wall thickness, $\mathrm{m}$ & $w$ & Random noise in AMV \\
\hline$M$ & Metabolic rate & $x$ & Thermal sensation state \\
\hline$\dot{m}$ & Air mass flow rate, $\mathrm{Kg} / \mathrm{s}$ & $y$ & Observed mean vote of thermal sensation \\
\hline
\end{tabular}

\section{Introduction}

Reducing building energy consumption and providing a better indoor thermal condition not only improves the environmental quality due to reduced emission rate, but also keeps people more productive at work and away from building health related problems. Current building control algorithms are mainly rule based (if-then-else based rules) and thus the performance of a large number of buildings heavily depends on the experience of building managers. In recent years, various advanced control techniques, such as fuzzy logic control [1]-[2], agent-based intelligent control [3], neural network control [4], optimal control [5], and model predictive control [6]-[19] have emerged in building control applications. 
In particular, model predictive control has gained increasing popularity in utilizing passive or active thermal storage to save energy [7]-[9]. With weather predictions and occupancy schedules, free cooling at night was used in cooling applications and night setback was adopted for heating applications [7][8]. Peak-load shifting was implemented in MPC to save electricity cost by taking advantage of the time-of-day rate of electricity price [9]. MPC was also applied to determine optimal temperature set-points at the top level of a hierarchical control, for which simple PID or on-off controllers were used for lower-level components such as fans, heating/cooling coils and thermal storage tanks [7]. Alternatively, low level components could be directly managed by a MPC to achieve a higher level of efficiency [11].

While on one hand energy saving is important, on the other hand, occupant thermal comfort plays a key role in the control of Heating, Ventilating and Air Conditioning (HVAC) systems for low-energy buildings. A large number of the existing control algorithms were designed such that the neutral temperature was achieved based on the Fanger's thermal comfort model. Alternatively, the so-called effective temperature, which is a combination of the indoor temperature and relative humidity, could be used as the index for thermal comfort [10]. Though the existing HVAC control algorithms seldom directly optimize a PMV index (or use it as a constraint), a numerical study demonstrated that using the PMV in defining the thermal comfort constraint in a MPC could reduce energy consumption and improve thermal comfort, compared to utilizing a comfort zone from a psychrometric chart [10].

However, direct incorporation of the PMV in a MPC design for HVAC systems could pose practical implementation challenges. The calculation of PMV involves iteration, which could raise computation concerns, especially for MPC which is known to be computation intensive. Past work tried to approximate the PMV with a neural network model [4][12][20] or with a linearized parameterization model [21]. In addition, most buildings typically do not have sensors to continually measure humidity, air velocity and mean radiant temperature. Even though for laboratory facilities where the aforementioned environmental sensing data are available, occupant clothing insulation and activity levels, which could vary with respect to time and vary among occupants, are seldom monitored continually and individually. Assuming a uniform and constant clothing level for occupants could cause errors in predicting occupant thermal sensations [11].

In this study, the MPC problem formulation used a data-driven dynamic thermal sensation (DTS) model developed using occupants' feedback on thermal perceptions [22]. A distinctive feature of this DTS model lies in 
that the time-varying offset parameter of the proposed Wiener-logistic model can be estimated through an extended Kalman Filter (EKF) using real-time occupant votes to capture the variability of thermal sensation due to environmental or occupant-associated changes. Rather than assuming that a "PMV sensor" exists [10], this study assumed that occupants act as a sensor for indoor thermal comfort and there exists a feedback channel for occupants to provide their thermal sensation votes to the controller. Field studies showed that there could be a discrepancy between Fanger's PMV and occupants' AMV [23]. Furthermore, occupants' awareness of opportunities to control their environment could affect their perceptions of thermal comfort [24], and occupants did often express their wishes to intervene automated control systems [25]. Though under the current building environment, it might not be practical for an individual occupant to directly control HVAC systems to achieve a personalized thermal environment, it is reasonable to assume that there is a feedback channel for occupants to communicate their thermal sensation perceptions to the controller.

Replacing Fanger's PMV by such a dynamic thermal sensation model in the MPC formulation enables the proposed MPC design for HVAC systems to adapt to uncertainties and variations associated with occupants' thermal perceptions. In addition, a chance-constrained MPC was also developed using the DTS model, which provides an opportunity for the controller to adjust the probability level of violation of thermal comfort to achieve a balance between energy consumption and thermal comfort.

\section{Models}

This section presents a data-driven dynamic thermal sensation model and a building model, which were used in the performance evaluation of the proposed MPCs.

\subsection{Experiments with human subjects}

Chamber experiments with human subjects were approved by the Institutional Review Board at the Pennsylvania State University (IRB \# 41077). The experiments were conducted in a climate chamber with its dimension shown in Fig. 1. The chamber was divided into two identical rooms and each room has its own HVAC unit. A HOBO U12 data logger was positioned in the middle of each room to measure air temperature and relative humidity. The mean radiant temperature of each room was measured by the BlackGlobe Temperature Sensor for Heat Stress 
(BlackGlobe) mounted at the same position as the HOBO U12 data logger. In the experiments, all environmental parameters were controlled to be the same for both rooms of the climate chamber, and both rooms were used at the same time to provide enough space for all participants. Therefore, the chamber can be viewed as a single virtual room and subject votes from the two rooms were not differentiated.

The experiments were conducted in February, with outdoor temperature around $5^{\circ} \mathrm{C}$ to $7^{\circ} \mathrm{C}$, and outdoor humidity level around $50 \%$. There were two sessions and each session lasted $2.5 \mathrm{hrs}$. In the first session, the room temperature was initially set at $21^{\circ} \mathrm{C}$ for 50 minutes, then raised to $30^{\circ} \mathrm{C}$ for another 50 minutes, and reduced back to $21^{\circ} \mathrm{C}$ again for the rest of the first session. The temperature of the second session started at $30^{\circ} \mathrm{C}$ for 50 minutes, then reduced to $23^{\circ} \mathrm{C}$ for another 50 minutes, and back to $30^{\circ} \mathrm{C}$ for the rest of the session. The available humidifier was not used to mimic the indoor environmental conditions typical for buildings without humidification. When the indoor air was heated to $21^{\circ} \mathrm{C}-30^{\circ} \mathrm{C}$, the resulting relative humidity of the chamber varied between $15 \%-25 \%$, with an average of $21 \%$. Since the experiments were designed to model how the changes of ambient temperature would affect occupants' thermal sensation, other inputs such as air velocity and occupants' activity level and clothing insulation were kept almost constant since 1) it would otherwise explode the number of experimental conditions, and 2) air temperature is typically the only control knob for low-energy buildings. Key environmental and occupant parameters of the chamber experiments are summarized in Table 1.

There were a total of 13 participants, including college and graduate students, who completed both sessions of the chamber experiments. The weight, height, age and gender of each participant were recorded to estimate each individual's basal metabolic rate. Clothing level of each subject was also recorded with detailed description. During the experiments, participants were allowed to work on laptops, read, and write in their sitting positions, and thus the activity level of each participant was considered sedentary. All participants were asked to write down their thermal sensation votes according to the ASHRAE 7-point thermal sensation scales every 5 minutes during a 20min time period immediately after the initial change of air temperature set-point, and then every 10 minutes for the rest of the session time.

\subsection{A data-driven dynamic thermal sensation model}

Based on the chamber experiments, a data-driven dynamic thermal sensation model was derived to describe occupants' thermal sensation variations due to changes of indoor air temperature as follows: 


$$
\begin{aligned}
& x(t+1)=0.798 \cdot x(t)+0.0610 \cdot x(t-1)+T_{a}(t)-0.883 \cdot T_{a}(t-1)+e(t) \\
& y(\mathrm{t})=\frac{3.033}{\exp [-0.558 \cdot(x(t)-7.931)+8.166]+1}+d(t)+v(t)
\end{aligned}
$$

This state-space model has a Wiener structure with a linear dynamic equation (1) and a logistic nonlinear output equation (2), where $x$ denotes the thermal sensation state, $T_{a}$ denotes the ambient air temperature input with the unit of Celsius, and $y$ denotes the observed/actual mean vote of thermal sensation. The process noise and measurement noise are denoted by $e$ and $v$, respectively. The sampling time of the discrete time dynamic model is $5 \mathrm{~min}$. The logistic output function in (2) has a constant regressor mean $(r=7.931)$, output coefficient $(a=3.033)$, dilation $(b=0.558)$, translation $(c=-8.166)$, but with a time varying offset parameter $d(t)$. The constant model coefficients in (1-2) and a nominal value for the offset parameter $d=-0.994$ were obtained by running a nonlinear least-squares algorithm over data collected from the chamber experiments.

In (1), the air temperature $T_{a}$ is modeled as the sole control input. We assume that the aggregate effect of other significant factors such as relative humidity, air velocity, mean radiant temperature and occupant-related factors is captured by the offset parameter $d(t)$ in (2). Additional thermal or non-thermal factors are considered as process and sensor noise.

When indoor environmental conditions or occupant clothing and activity level deviate from the nominal condition conducted in the chamber experiments, a new offset parameter $d$ is re-estimated using EKF [26] to effectively correct the model prediction of thermal sensation. Essentially, the offset parameter $d$ is modeled as a (discrete time) Wiener process as follows:

$$
d(k+1)=d(k)+e_{d}(k)
$$

where the parameter process noise $e_{d}$ is assumed to have zero mean and nonzero variance $Q_{d}$. Define an augmented state variable $x_{A}(k)$ consisting of the original state variable $x(k-1), x(k)$, and the time-varying offset parameter $d(k), x_{A}(k)=\left[\begin{array}{lll}x(k-1) & x(k) & d(k)\end{array}\right]^{T}$. Then an EKF is applied to estimate the augmented state variable, i.e., estimating the thermal sensation state and the time-varying offset parameter $d$ simultaneously (see the Appendix). This dynamic thermal sensation model was presented before in the authors' paper [22]. 


\subsection{Building thermal transport model}

The thermal transport model for the chamber was derived using a commonly-used RC thermal network based on heat balance [27]-[28]. For a RC thermal network, each wall or zone is abstracted as a node. Each node is then modeled as a thermal capacitance (thermal mass) with its temperature representing the state of the node. The network is formed by connecting all nodes with thermal resistors. The capacitances and resistances can be estimated based on the geometry and material properties of the building under study.

It is important to notice that the thermal network model is a simple representation of actual building environment and its mechanical system. Building network models are widely used and recently improved for their capabilities to accurately predict energy consumption in buildings [29]. These models are popular not only for their capabilities to accurately predict energy consumption in actual buildings, but also for their simplicity, allowing testing of different control algorithms prior to their deployment and further testing in more complicated scenarios [30]. In the present

study, several important assumptions are made for the network model including negligible internal radiative fluxes, which are applicable to spaces without windows and radiative cooling/heating systems, such as chilled ceiling or heating radiators [22]. Therefore, the thermal network model treats the conductive and convective heat transfer as well as thermal storage, but it neglects the radiative heat transfer. It is important to notice that this assumption also implies that the mean radiant temperature, used in thermal comfort models, is equal to the air temperature in the studied space. Our experimental study showed that this assumption is correct for a space with well-insulated walls, without windows or radiative cooling/heating systems, such as the studied chamber environment [22]. Furthermore, the thermal network model assumes no recirculation of the air supplied to the space, so the total amount of energy required for cooling or heating the studied space is overestimated, but the actual trends and relative differences are following performance in actual spaces. Overall, the simplifications used in the thermal network model result in heat flux values that deviate from actual systems, but represent correct trends and orders of magnitude, so they provide a good representation of an actual system to test the differences in performance of controls algorithms. Our future study will account for a realistic mechanical system representation, such as EnergyPlus software [31].

Fig. 2 shows the RC thermal network for a single-room chamber, for which the roof and floor are ignored. There are 5 nodes in the system. Node 1 represents the room air and other nodes represent the four single-layered walls of 
the room. Let $T_{i}(i=1, \cdots, 5)$ denote the temperature of the node $i=1, \cdots, 5$, respectively. Let $T_{O}$ denote the outdoor temperature. Without loss of generality, it is assumed that wall 2 faces outside and the rest of the walls are connected to adjacent rooms (not shown in the figure) with temperature denoted by $T_{N}$.

The capacitance of each node, $C_{i}(i=1, \ldots, 5)$, depends on the lumped mass of the node and specific heat of the wall material. Each lumped thermal resistance between each pair of nodes is estimated based on different ways of heat transfer given as follows: 1) for conduction within the wall, $R_{\text {cond }}=\frac{l_{\text {wall }}}{2 k A_{w}}$, where $l_{\text {wall }}$ denotes the thickness of the wall, $k$ is the conduction coefficient of the wall material, and $A_{w}$ is the area of the wall; 2) for convection between a wall surface and air, $R_{\text {conv }}=\frac{1}{h A_{w}}$, where $h$ denotes the convection coefficient. In this study, all walls are assumed to be the same, and thus the thermal resistance between each pair of nodes (i, $\mathrm{j}), R_{i j}$, is assumed to be the same and it is computed as follows,

$$
R_{i j}=R_{\text {cond }}+R_{\text {conv }}=\frac{l}{2 k A_{w}}+\frac{1}{h A_{w}}
$$

The air change rate is set at $1 \mathrm{ACH}$. The heat exchange due to ventilation can be calculated as,

$$
q_{\text {vent_flow }}=\dot{m}\left(h_{\text {out }}-h_{\text {in }}\right)=\dot{m}\left\{\left[c_{p a} \cdot T_{o}+W_{\text {out }}\left(c_{p w} \cdot T_{o}+h_{w e}\right)\right]-\left[c_{p a} \cdot T_{1}+W_{\text {in }}\left(c_{p w} \cdot T_{1}+h_{w e}\right)\right]\right\}
$$

Where $q_{\text {vent_flow }}$ denotes the ventilation heat flow, $\dot{m}$ denotes the air mass flow rate and can be calculated based on the volume of the room and the air change rate, $h_{\text {out }}$ denotes the outdoor specific enthalpy of moist air, $h_{\text {in }}$ denotes the indoor specific enthalpy of moist air, $c_{p a}$ denotes the specific heat of air, $c_{p w}$ denotes the specific heat of water vapor, $T_{o}$ denotes the outdoor temperature, $T_{1}$ denotes the indoor temperature (temperature of node 1$), W_{\text {out }}$ denotes the outdoor humidity ratio, $W_{i n}$ denotes the indoor humidity ratio, and $h_{w e}$ denotes the evaporation heat of water at 0 Celsius. It is important to note that this model assumes no recirculation, so the entire airflow rate represents outdoor airflow into the space. This is an assumption introduced to simplify the thermal network model.

Humidity ratio $W$ can be calculated as, 


$$
W=\frac{R H \cdot W_{s}}{1+(1-R H) W_{s} / 0.6220}
$$

where $R H$ denotes relative humidity, $W_{s}$ denotes saturation humidity ratio, which can be looked up for specific air temperatures. By (5), the indoor and outdoor humidity ratio $W_{\text {in }}$ and $W_{\text {out }}$ can be computed respectively.

Let $G_{\text {int }}$ denote the internal gain. Then based on energy conservation, the temperature of each node,

$T_{i}, i=1, \cdots, 5$, satisfies the following state-space model,

$$
\begin{aligned}
& C_{1} \frac{d T_{1}}{d t}=\frac{T_{2}-T_{1}}{R_{21}}+\frac{T_{3}-T_{1}}{R_{31}}+\frac{T_{4}-T_{1}}{R_{41}}+\frac{T_{5}-T_{1}}{R_{51}}+q_{\text {vent }_{-} \text {flow }}+G_{\text {int }}+u \\
& C_{2} \frac{d T_{2}}{d t}=\frac{T_{1}-T_{2}}{R_{21}}+\frac{T_{O}-T_{2}}{R_{2 O}} \\
& C_{3} \frac{d T_{3}}{d t}=\frac{T_{1}-T_{3}}{R_{31}}+\frac{T_{N}-T_{3}}{R_{3 N}} \\
& C_{4} \frac{d T_{4}}{d t}=\frac{T_{1}-T_{4}}{R_{41}}+\frac{T_{N}-T_{4}}{R_{4 N}} \\
& C_{5} \frac{d T_{5}}{d t}=\frac{T_{1}-T_{5}}{R_{51}}+\frac{T_{N}-T_{5}}{R_{5 N}}
\end{aligned}
$$

Where the control input $u$ denotes the heating $(u>0)$ or cooling $(u<0)$ flow.

Define $T=\left[\begin{array}{llll}T_{1} & T_{2} & \cdots & T_{5}\end{array}\right]^{T}$ (in Kelvin). Consider a constant internal gain $G_{\text {int }}=500$ watt and a neighboring room temperature of 25 Celsius. By discretization of the continuous time model with a $15 \mathrm{~min}$ sampling time, the resulting discrete-time building thermal transport model can be written in the following compact state-space form,

$$
\begin{aligned}
& T(t+1)=A T(t)+B_{1} T_{O}(t)+B_{2} T_{N}(t)+B_{3}\left[u(t)+G_{\text {int }}+q_{\text {vent }- \text { flow }}(t)\right] \\
& T_{a}(t+1)=C T(t+1)-273.15
\end{aligned}
$$


Where the unit of the room air temperature $T_{a}$ is Celsius, and numerical values of the matrices $A, B_{1}, B_{2}, B_{3}$ and $C$ can be calculated based on the geometry and material properties of the chamber given in Table 2 and their values are given as follows:

$$
\begin{aligned}
& A=\left[\begin{array}{lllll}
0.1843 & 0.2026 & 0.2026 & 0.2026 & 0.2026 \\
0.0051 & 0.9802 & 0.0014 & 0.0014 & 0.0014 \\
0.0051 & 0.0014 & 0.9802 & 0.0014 & 0.0014 \\
0.0051 & 0.0014 & 0.0014 & 0.9802 & 0.0014 \\
0.0051 & 0.0014 & 0.0014 & 0.0014 & 0.9802
\end{array}\right], \\
& B_{1}^{T}=\left[\begin{array}{lllll}
0.0014 & 0.0106 & 0 & 0 & 0
\end{array}\right], \\
& B_{2}^{T}=\left[\begin{array}{lllll}
0.0041 & 0 & 0.0106 & 0.0106 & 0.0106
\end{array}\right], \\
& B_{3}^{T}=\left[\begin{array}{lllll}
0.0034 & 0 & 0 & 0 & 0
\end{array}\right] \\
& C=\left[\begin{array}{lllll}
1 & 0 & 0 & 0 & 0
\end{array}\right]
\end{aligned}
$$

\section{Control Formulation}

Two types of MPC designs using the dynamic thermal sensation model (1-2) were developed to minimize energy consumption while maintaining occupant thermal comfort. At each time instant $t$, the MPC solves a finite-horizon constrained minimization of energy consumption to determine the optimal heating/cooling trajectory based on the prediction of indoor conditions (from the building model) and prediction of occupants' actual mean vote (from the DTS model). Only the first step of the control trajectory is implemented at the current time instant $t$ and the procedure is repeated at the next time instant. For the simulations conducted in this study, outdoor weather conditions were assumed to be known (historical data were used), based on which prediction of indoor air temperature was computed using the building thermal transport model (10-11). Due to the considered process noise and sensor noise in the DTS model (1-2), the observed occupants' AMV is assumed to be a random variable.

In the first MPC, the thermal comfort constraint is specified in terms of the DTS model prediction for AMV and the controller assumes that the prediction is correct under certainty equivalence, i.e., the disturbance/noise is 
assumed to take its expected value. This MPC is referred to as Certainty Equivalence MPC, denoted as CEMPCDTS. In the second MPC, a stochastic thermal comfort constraint is defined, which requires that the probability of AMV within a specified bound is larger than a preset number. This MPC is referred to as Chance-Constrained $M P C$, denoted as $C C M P C-D T S$. A deterministic approximation of the chance constraint was adopted in this paper, which leads to a tighter bound on thermal comfort than that used in CEMPC.

Performance of the CEMPC-DTS and CCMPC-DTS were compared to a MPC using Fanger's PMV as the thermal comfort index (denoted as MPC-PMV) and a rule-based PI control (denoted as RuleBased-PI) that represents the current control practice for HVAC systems. Control formulations of the three MPC designs are given in Section 3.1 and details on the RuleBased-PI are given in Section 3.2.

\subsection{MPC formulations}

The general MPC formulation is described as follows.

Cost: $\min _{U_{t}} J_{t}:=\sum_{k=1}^{N}[u(t+k \mid t)]^{2}+\lambda \sum_{k=1}^{N}[q(t+k \mid t)]$

This cost function is subject to the following models for building thermal transport and thermal sensation for building occupants.

Building thermal transport model:

$T(t+k+1 \mid t)=A T(t+k \mid t)+B_{1} T_{O}(t+k \mid t)+B_{2} T_{N}(t+k \mid t)+B_{3}\left[u(t+k \mid t)+G_{\text {int }}(t+k \mid t)+q_{\text {vent } \_ \text {flow }}(t+k \mid t)\right]$

$T_{a}(t+k+1 \mid t)=C T(t+k+1 \mid t)-273.15$

Thermal sensation model:

$T S(t+k \mid t)=f\left(T_{a}(t+k \mid t), T_{a}(t+k-1 \mid t), \ldots\right)$

Constraint on thermal comfort:

$y_{\min }-q(t+k \mid t) \leq T S(t+k \mid t) \leq y_{\max }+q(t+k \mid t)$ 
$q(t+k \mid t) \geq 0$

Constraint on cooling and heating power:

$u_{\min } \leq u(t+k \mid t) \leq u_{\max }$

where $U_{t}=[u(t+1 \mid t), \cdots, u(t+N \mid t)]$ denotes the control input trajectory along the prediction horizon, and $N$

denotes the length of the prediction horizon. The cost function $J_{t}$ is defined as the $L_{2}$ norm of the control input $u(t+k \mid t)$ plus the $L_{1}$ norm of the slack variable $q(t+k \mid t)$ for satisfying the thermal comfort constraint. A very large number $\lambda$ is chosen to heavily penalize the violation of thermal comfort. TS represents thermal sensation in a generic model (16), which will be substituted by different thermal comfort models in the subsequent subsections. A soft constraint (17) is considered for maintaining the thermal sensation within the bounds $y_{\min }$ and $y_{\max }$ using the slack variable $q$ to avoid infeasibility in solving the resulting MPC. The control input is subject to constraint (19) with the maximum cooling power denoted by $u_{\min }\left(u_{\min }<0\right)$ and the maximum heating power denoted by $u_{\max }\left(u_{\max }>\right.$ $0)$.

\subsubsection{Certainty Equivalence MPC based on DTS model (CEMPC-DTS)}

In CEMPC-DTS, the thermal sensation equation (16) is computed using the DTS model expressed in (1-2) under the assumption that the noise $e(t)$ and $v(t)$ take their respective expected values. In this study, the process noise $e(t)$ and sensing noise $v(t)$ are assumed to be Gaussian with zero mean, i.e., $E[e(t)]=0$ and $E[v(t)]=0$. As a result, the following thermal sensation prediction is used to substitute (16) in CEMPC-DTS:

$$
\begin{aligned}
& \hat{x}(t+k+1 \mid t)=0.798 \cdot \hat{x}(t+k \mid t)+0.0610 \cdot \hat{x}(t+k-1 \mid t)+T_{a}(t+k \mid t)-0.883 \cdot T_{a}(t+k-1 \mid t) \\
& \hat{d}(t+k \mid t)=\hat{d}(t+k-1 \mid t) \\
& \hat{y}(t+k \mid t)=\frac{3.033}{\exp [-0.558 \cdot(\hat{x}(t+k \mid t)-7.931)+8.166]+1}+\hat{d}(t+k \mid t) \\
& T S(t+k \mid t)=\hat{y}(t+k \mid t)
\end{aligned}
$$


Note that the coefficient values in (20-22) correspond to a $5 \mathrm{~min}$ sampling time and re-discretization using a $15 \mathrm{~min}$ sampling time was conducted in the controller simulation.

\subsubsection{Chance Constrained MPC based on DTS model (CCMPC-DTS)}

CCMPC-DTS considers the following probabilistic (chance) constraint for thermal comfort:

$\operatorname{Prob}\left\{y_{\min } \leq T S(t+k \mid t) \leq y_{\max }\right\} \geq 1-\alpha$

Where $\alpha$ denotes a predefined small percentage. Assuming a Gaussian distributed process/sensor noise with zero mean, the chance constraint (24) can be approximated by a deterministic constraint [32] as follows:

$y_{\min }+\Delta y \leq \hat{y}(t+k \mid t) \leq y_{\max }-\Delta y$

$\Delta y=\beta \Omega^{1 / 2}$

Where $\hat{y}(t+k \mid t)$ denotes the prediction for AMV given by the DTS model (20-22), $\beta=\sqrt{2} \operatorname{erf}^{-1}(1-2 \alpha)$ with

$\operatorname{erf}^{-1}$ denoting the inverse of the standard error function $\operatorname{erf}(x)=\frac{2}{\sqrt{\pi}} \int_{0}^{x} e^{-u^{2}} d u, \Omega$ corresponds to the measurement prediction covariance in (A-11) (which is a scalar here due to the single output variable) and it can be computed using EKF.

By further softening the constraint using the slack variable $q$ in CCMPC-DTS, thermal constraint (17) is replaced by

$$
\left(y_{\min }+\Delta y\right)-q(t+k \mid t) \leq \hat{y}(t+k \mid t) \leq\left(y_{\max }-\Delta y\right)+q(t+k \mid t)
$$

Compared to the constraint used in CEMPC-DTS, the constraint in CCMPC-DTS offers two advantages: 1) a tunable probability of constraint violation $\alpha$, and 2) a tighter thermal comfort bound to improve thermal comfort.

\subsubsection{MPC based on PMV model (MPC-PMV)}


In the MPC-PMV formulation, Fanger's PMV model is used to implement (16) for $T S(t+k \mid t)$,

$$
T S(t+k \mid \mathrm{t})=P M V\left(T_{a}(t+k \mid t), T_{m r}, R H, V_{a}, C L O, M\right)
$$

Where the air temperature prediction $T_{a}(t+k \mid t)$ is obtained from the building model (14-15), the mean radiant temperature $T_{m r}$ is assumed to be the same as the air temperature, and the relative humidity $R H$, air velocity $V_{a}$, occupants' clothing insulation level $C L O$ and metabolic rate (activity level) $M$ at time $(t+k)$ are assumed to be known.

\subsection{RuleBased-PI control}

In this control strategy, the thermal comfort constraint is defined by specifying heating and cooling set points, denoted by $S P_{\text {heating }}$ and $S P_{\text {cooling }}$ respectively, with a buffer to prevent short cycling. The actual values of these set points are chosen by building managers and they vary by seasons and climate regions. If the indoor temperature is low $\left(T_{a}<S P_{\text {heating }}-\right.$ buffer $)$, a PI control is applied to increase the indoor temperature to the heating set point $S P_{\text {heating }}$. If the indoor temperature is high $\left(T_{a}>S P_{\text {cooling }}+\right.$ buffer $)$, a PI control is applied to cool the indoor air temperature to the cooling set point $S P_{\text {cooling }}$. The PI control with a low-pass filter used in this paper is given as follows:

$u_{-}(k)=K_{p} \cdot \operatorname{err}(k)+K_{I} \cdot \Delta T \cdot \sum_{i=0}^{k} \operatorname{err}(i)$

$u(k)=0.1 \cdot u_{-}(k)+0.9 \cdot u(k-1)$

Where err denotes the error between the heating set point $S P_{\text {heating }}$ (or cooling set point $S P_{\text {cooling }}$ ) and indoor air temperature $T_{a}, K_{p}$ and $K_{I}$ are proportional and integral gain, respectively, and $\Delta T$ is the sampling time.

\section{Simulation Results}

\subsection{Simulation platform}


Performance of the two DTS-based MPC, CEMPC-DTS and CCMPC-DTS, was compared to the MPC-PMV and Rule-based PI through simulations. Here, the occupants' AMV is simulated using the PMV plus a random white noise $w$,

$$
A M V(k)=P M V(k)+w(k)
$$

Where the random noise $w$ is assumed to follow a Gaussian distribution. When $w$ has a zero mean $E[w]=0$, we assume that the PMV is an accurate model to describe the occupants' AMV without bias. When $w$ has a nonzero mean, i.e., $E[w] \neq 0$, we assume that the PMV is biased in evaluating AMV, noting that PMV was reported to underestimate or overestimate thermal sensation in field surveys [22],[23]. In the remaining of this paper, the output of the virtual occupant model (31) is referred to as virtual occupants'feedback.

Fig. 3 shows the block diagram for the DTS model based MPC controller (either CEMPC-DTS or CCMPCDTS) used in simulations. It is assumed that occupants' feedback on thermal sensation is available to be used by the MPC design. At each time step of the simulation, the observed occupants' AMV is first passed to an extended Kalman Filter to estimate the state variable $\hat{x}(t+k \mid t)$ and the offset parameter $\hat{d}(t+k \mid t)$ simultaneously (details given in the Appendix). These two parameters are then fed into the DTS model to generate the prediction of AMV $\hat{y}(t+k \mid t)$ along the optimization horizon. By solving the corresponding MPC optimization (CEMPC or CCMPC), the optimal heating or cooling flow is determined and then fed into the building model to compute the resulting room temperature.

For the MPC-PMV controller, there is neither an "EKF" block nor feedback of occupants' thermal sensation to the MPC-PMV control. Among the six input variables to the PMV model, the room air temperature is predicted by the building model (13-14), the mean radiant temperature is set to be the same as the air temperature, the air velocity and occupant activity level are set to the values used in a chamber experiment [22] (see Table 1), and the indoor relative humidity and clothing insulation levels are designed to start from the nominal values given in Table 1 and then follow the trajectories shown in Fig. 4 and Fig. 5, respectively.

The simulation time step $\Delta T$ is set to be $15 \mathrm{~min}$. The MPC optimization horizon is chosen to be $N=20$ sampling intervals equivalent to $5 \mathrm{hrs}$. The bounds on thermal sensation are set to be $y_{\min }=-0.5$ and $y_{\max }=0.5$. The control 
input is constrained by $u_{\min }=-5000$ (for 5 kilowatts max cooling power) and $u_{\max }=5000$ (for 5 kilowatts $\max$ heating power) respectively. In the RuleBased-PI, parameters are set to be $S P_{\text {heating }}=24$ Celsius, $S P_{\text {cooling }}=26$ Celsius, buffer $=1$ Celsius, $K_{p}=2000$ and $K_{I}=100$.

\subsection{Environmental and occupant parameters}

To evaluate the control performance, simulations in this study used two different sets of outdoor weather conditions shown in Fig. 6. In Fig. 6, the subplots show the outdoor temperature and relative humidity history data collected at State College, PA for 3 days from 03/09/2013 to 03/11/2013 in March and 3 days from 09/10/2013 to 09/12/2013 in September. The indoor relative humidity trajectory used in the simulations (shown in Fig. 4) is designed to start at a nominal value of $21 \%$ in the first day followed by a sinusoidal curve ranging from $21 \%$ to $80 \%$ with a period of 24 hours for two days. This indoor relative humidity parameter is designed to follow such a trajectory so that the proposed controllers can be tested in very low and very high humidity conditions within a relative short simulation time period. The average clothing level of occupants is plotted in Fig. 5, which is set to $C L O=0.71$ for the first two days, raised to $C L O=0.85$ at the beginning of the third day and then maintained at this value for the rest of the day. The initial values of the relative humidity and average clothing level were chosen from operating conditions of the chamber experiment [22] conducted to obtain nominal coefficients of the DTS model (12).

To generate the virtual occupant feedback on thermal sensation, the noise $w$ that is added to the PMV in (31) is chosen to be a Gaussian noise with a standard deviation of 0.08 with three different mean values: 1) $E[w]=0$, used to simulate the scenario where there is no discrepancy between the PMV and AMV; 2) $E[w]=0.2$, used to simulate the scenario where the PMV underestimates the AMV, and 3) $E[w]=-0.2$, used to simulate the scenario where the PMV overestimates the AMV.

\subsection{Performance metrics}


Three metrics are used to evaluate the control performance. The first one is total Energy Consumption (E), which computes the integration of control input (heating/cooling power) over time. The second one is Violation Ratio (VR), which is defined as the ratio of the time during which the AMV generated by the Virtual Occupants falls outside the thermal sensation bounds over the total simulation time ( 3 days). This metric indicates how frequently the constraint on thermal comfort is violated, but it does not provide information on how far the AMV is away from the specified bounds when the constraint is violated. The third metric, Integration of Violation (IOV), computes the area between the thermal sensation bound ( 0.5 or -0.5 depending on cooling or heating application) and the AMVs that fall outside the bound.

\subsection{Results and discussions}

Considering two sets of weather conditions, three different expected values for the Gaussian noise $w$ in simulating virtual occupants, and four controllers, there are a total of 24 cases being evaluated. Table 3 summarizes the performance statistics, which are averages of 20 simulation runs for each case. Simulation plots are presented in Fig. 7 - Fig. 10 for two representative scenarios: Scenario $I$ corresponds to $E[w]=0$, and Scenario II corresponds to $E[w]=-0.2$, both for the time period in March. Due to the limited space, simulation plots for other cases are omitted here.

\subsubsection{RuleBased-PI versus MPC}

Table 3 shows that when $E[w]=0$, all three MPC algorithms use less amount of energy than the RuleBased-PI while providing better thermal comfort (lower IOV). When $E[w] \neq 0$, at least one of the three MPC algorithms consumes less energy with lower (or comparable) IOV than the RuleBased-PI. Fig. 7 - Fig. 8 shed some light on the underlying cause for energy savings with improved thermal comfort for MPC designs. The room temperature profiles given in Fig. 7 show that the RuleBased-PI tries to regulate the room temperature to the heating set point at 24 Celsius, irrespective of the changes in relative humidity and occupant clothing levels. In contrast, room temperatures controlled by the three MPC designs are adjusted to adapt to the changes in RH and clothing levels. For example, in the second day the room temperatures controlled by MPCs are observed to follow a roughly flipped pattern of the humidity curve to maintain the same level of thermal sensation. In the third day, due to the increased 
clothing insulation level, indoor temperatures are reduced to a lower value to save energy consumption (see also Fig. 8 for control input trajectories). Fig. 9 shows that for all three MPC designs, AMV is closely regulated to be constant except the times when changes of RH and clothing level occur, but the AMV for the RuleBased-PI varies a lot.

\subsubsection{MPC-PMV versus CEMPC-DTS}

Scenario I (March, $E[w]=0$ ) represents the case where the PMV is assumed to be an accurate prediction for occupants' AMV. Fig. 7(a) shows that the difference between the two air temperature trajectories controlled by the MPC-PMV and CEMPC-DTS is very limited. The control input trajectories of the two controllers (shown in Fig. 8(a)) are also very close to each other except for the short periods when the changes in RH and clothing level occur. These discrepancies are mainly due to the learning error of the DTS model. Statistics from Table 3 also confirm this observation, with the CEMPC-DTS having a slightly higher energy consumption or IOV than the MPC-PMV.

Scenario II ( March, $E[w]=-0.2$ ) represents the case where PMV overestimates the AMV, i.e., the occupants feel colder than predicted by the PMV. As a result, the room temperature of the CEMPC-DTS is controlled at a higher value (close to 1 Celsius higher) with more energy consumption than the MPC-PMV (shown by Fig. 7(b) and Fig. 8(b)) to regulate the AMV at -0.5 (shown by Fig. 9(b)). Without feedback from occupants, the AMV resulted from the MPC-PMV is lower than the thermal comfort threshold of -0.5 scale.

When $E[w]=0.2$ during the 3day period in March (figures not shown due to the limited space), occupants feel warmer than predicted and thus less heating power is needed to save energy. Case 7 of Table 3 shows that the CEMPC-DTS has cut the energy consumption more aggressively than desired and thus leads to a higher IOV than the MPC-PMV. This indicates that a better balance between the energy saving and thermal comfort is needed, which has been investigated in the design of the CCMPC-DTS.

The cases for the September time period show similar trends as the March time period, as indicated by statistics from Table 3. When $E[w]=0$, the CEMPC-DTS has a similar level of energy consumption, VR and IOV to the MPC-PMV. When $E[w]=0.2$, occupants feel hotter than predicted by the PMV and thus demand more cooling 
(more energy consumption) to satisfy the thermal comfort constraint (reduced IOV). When $E[w]=-0.2$, occupants feel cooler than predicted by the PMV and thus less cooling power is needed to save energy.

For the completeness of information, Fig. 10 plots the offset parameter of the DTS model estimated by an EKF using occupants' feedback on thermal sensation. The variation of the offset parameter at the beginning of the first day is due to model calibration. Its variations at the second and third day show a clear adaptation to changes of the relative humidity and occupant clothing level.

\subsubsection{CEMPC-DTS versus CCMPC-DTS}

Table 3 shows that the thermal comfort violation ratio VR is often high for the deterministic control approaches (Rulebased-PI, MPC-PMV and CEMPC-DTS), even when $E[w]=0$. This implies that mean-vote based deterministic (or certainty-equivalent) controls are likely not able to meet the thermal comfort constraint if the variance of occupant perceptions of thermal sensation is large. The CCMPC-DTS provides an opportunity to directly specify the probability that thermal comfort constraint will be satisfied. In addition, the tighter bound on thermal comfort imposed by the deterministic approximation of the chance constraint helps to improve the thermal comfort level, though at the expense of a larger energy consumption. Table 3 shows that when $E[w]=0$, the CCMPC-DTS has the highest amount of energy consumption among all three MPC controllers, but with a significantly lower VR and IOV.

\subsubsection{Tuning $\alpha$ to achieve a balance between energy saving and thermal comfort}

To investigate the tradeoff between energy saving and comfort violation, the probability $\alpha$ of thermal constraint violation is varied in the CCMPC-DTS to generate the Pareto frontier, as shown in Fig. 11, for energy consumption versus thermal comfort violation when $E[w]=0$. It is also shown in Fig. 11 that the RuleBased-PI always stay upper right to the Pareto frontier, indicating that the CCMPC-DTS can reduce energy usage while improve thermal comfort at the same time compared to the RuleBased-PI. Corresponding to similar level of thermal comfort, the 
difference in energy consumption between the MPC-PMV and the CCMPC-DTS is around 5\% - 8\%. This difference is due to the noise variance used in simulating virtual occupants' feedback.

\section{Conclusion and Future Work}

In this study, two model predictive control strategies using a data-driven dynamic thermal sensation model were developed as complementary design tools to the PMV-based MPC control for optimizing indoor thermal comfort and energy saving. The proposed chance-constrained MPC was designed to allow specifying the probability of violation of thermal comfort constraint, in order to achieve a balance between energy saving and thermal comfort. Simulation results demonstrated that an MPC based on occupant feedback can be expected to produce better thermal comfort and energy outcomes than an MPC based on a theoretical thermal comfort proxy such as PMV.

This study assumes that there is a feedback channel for occupants to communicate their thermal perceptions to the control system, which is lacking in the current building control systems. Experimental testing and evaluation of the proposed DTS based MPC design algorithms in a chamber environment will be conducted in the future work.

\section{Acknowledgement}

This project is funded by NSF grant EFRI-1038264/EFRI-1452045. The chamber experiment was approved by the IRB at Penn State University (IRB \#41077). We would like to thank Dr. Moshood Fadeyi, Dr. Mohammad Heidarinejad, Dr. Yang-Seon Kim and Dr. Mingjie Zhao for their help with the chamber experiment and their valuable comments. We also would like to thank the anonymous reviewers for their insightful reviews.

\section{Appendix: An Extended Kalman Filter for estimating thermal sensation state and time-varying offset parameter simultaneously}

Consider the dynamic thermal sensation model (1-2),

$x(k+1)=f_{1} x(k)+f_{2} x(k-1)+g_{1} T_{a}(k)+g_{2} T_{a}(k-1)+e(k)$ 
$y(k)=\frac{a}{\exp [-c-b \cdot(x(k)-r)]+1}+d+v(k)$

where the model coefficients $f_{1}, f_{2}, g_{1}, g_{2}, a, b, c, r$ are constant and defined accordingly. We assume that the offset parameter $d$ is slowly time-varying and modeled as a (discrete time) wiener process as follows:

$d(k+1)=d(k)+e_{d}(k)$

where the parameter process noise $e_{d}$ is assumed to have zero mean and nonzero variance $Q_{d}$.

Define an augmented state variable $x_{A}(k)$ consisting of the original state variable $x(k), x(k-1)$, and the timevarying parameter $d(k)$,

$x_{A}=\left[\begin{array}{l}x_{A_{1}} \\ x_{A_{2}}\end{array}\right], x_{A_{1}}=\left[\begin{array}{c}x_{A_{1}}^{1} \\ x_{A_{1}}^{2}\end{array}\right]\left[\left[\begin{array}{c}x(k) \\ x(k-1)\end{array}\right], x_{A_{2}}(k) \square d(k)\right.$

Then the original thermal sensation model (A-1 and A-2) together with the parameter model (A-3) can be rewritten as follows,

$x_{A}(k+1)=F x_{A}(k)+G \circ T_{a}(k)+\varepsilon(k)$

$y(k)=h\left(x_{A}(k)\right)+v(k)$

where,

$G \circ T_{a}(k)=\left[\begin{array}{lll}g_{1} T_{a}(k)+g_{2} T_{a}(k-1) & 0 & 0\end{array}\right]^{T}, \varepsilon(k)=\left[\begin{array}{lll}e(k) & 0 & e_{d}(k)\end{array}\right]^{T}$,

$F=\left[\begin{array}{ccc}f_{1} & f_{2} & 0 \\ 1 & 0 & 0 \\ 0 & 0 & 1\end{array}\right]$

and the output nonlinear function satisfies,

$h\left(x_{A}(k)\right)=\frac{a}{\exp \left(-c-b\left(x_{A_{1}}^{1}(k)-r\right)\right)+1}+x_{A_{2}}(k)$.

The process noise $\varepsilon(k)$ and sensor noise $v(k)$ are assumed to be white noise and satisfy the following, 


$$
\begin{aligned}
& E[\varepsilon(k)]=0, E\left[\varepsilon(k) \varepsilon^{T}\left(k^{\prime}\right)\right]=Q(k) \delta_{k k^{\prime}} \\
& E[v(k)]=0, E\left[v(k) v^{T}\left(k^{\prime}\right)\right]=R(k) \delta_{k k^{\prime}}
\end{aligned}
$$

It is assumed that the initial estimate $\hat{x}_{A}(0 \mid 0)$ is uncorrelated with the process noise and sensor noise sequences. Assume that one has the estimate $\hat{x}_{A}(\mathrm{k} \mid \mathrm{k})=\mathrm{E}\left[x_{A}(k) \mid Z^{k}\right]$ at time $k$, with the associate covariance matrix $P(k \mid k)$, where $Z^{k}$ denotes the statistics obtained at time $k$.

The predicted state at time $(\mathrm{k}+1)$ based on time $\mathrm{k}$ is obtained as follows,

$\hat{x}_{A}(k+1 \mid k)=F \hat{x}_{A}(k \mid k)+G \circ T_{a}(k)$

The state prediction covariance is updated as

$$
P(k+1 \mid k)=F P(k \mid k) F^{T}+Q(k)
$$

The predicted measurement for the 2nd-order Kalman filter is given by,

$$
\hat{\mathrm{y}}(\mathrm{k}+1 \mid \mathrm{k})=h\left(\hat{x}_{A}(\mathrm{k}+1 \mid \mathrm{k})\right)+\frac{1}{2} \operatorname{tr}\left[h_{x_{A} x_{A}}(\mathrm{k}+1) P(\mathrm{k}+1 \mid \mathrm{k})\right]
$$

and the measurement prediction covariance matrix is updated as

$$
S(\mathrm{k}+1)=h_{x_{A}}(\mathrm{k}+1) \mathrm{P}(\mathrm{k}+1) h_{x_{A}}^{T}(\mathrm{k}+1)+\frac{1}{2} \operatorname{tr}\left[h_{x_{A} x_{A}}(\mathrm{k}+1) P(\mathrm{k}+1 \mid \mathrm{k}) h_{x_{A} x_{A}}(\mathrm{k}+1) P(\mathrm{k}+1 \mid \mathrm{k})\right]+R(\mathrm{k}+1)
$$

where $h_{x_{A}}$ and $h_{x_{A} x_{A}}$ denote the Jacobian and Hessian of the nonlinear function $h$, respectively,

$$
\begin{aligned}
& h_{x_{A}}(\mathrm{k}+1)=\left.\left[\nabla_{x_{A}} h\left(x_{A}\right)\right]^{T}\right|_{x_{A}=\hat{x}_{A}(\mathrm{k}+1 \mid \mathrm{k})}, \\
& h_{x_{A} x_{A}}(\mathrm{k}+1)=\left.\left[\nabla_{x_{A}} \nabla_{x_{A}}^{T} h\left(x_{A}\right)\right]\right|_{x_{A}=\hat{x}_{A}(\mathrm{k}+1 \mid \mathrm{k})}
\end{aligned}
$$

\section{REFERENCES}

[1] A.I. Dounis, D.E. Manolakis, Design of a fuzzy system for living space thermal-comfort regulation, Applied Energy 69 (2001) 119-144. 
[2] J. Singh, N. Singh, J.K. Sharma, Fuzzy modeling and control of HVAC systems - A review, Journal of Scientific and Industrial Research 65 (2006) 470-476.

[3] K. Dalamagkidis, D. Kolokotsa, K. Kalaitzakis, G.S. Stavrakakis, Reinforcement learning for energy conservation and comfort in buildings, Building and Environment 42 (2006) 2686-2698.

[4] J. Liang, R. Du, Thermal comfort control based on neural network for HVAC application, in: Proc. of IEEE Conference on Control Applications, Toronto, Canada, 2005.

[5] J.E. Braun, Load control using building thermal mass, Journal of Solar Energy Engineering 125 (2003) 292-301.

[6] M. Zaheer-uddin, G.R. Zheng, Optimal control of time scheduled heating ventilation and air conditioning processes in building, Energy Conversion and Management 41 (2000) 49-60.

[7] Y. Ma, A. Kelman, A. Daly, F. Borrelli, Predictive control for energy efficient buildings with thermal storage: Modeling, simulation, and experiments, IEEE Control Systems Magazine 32 (2012) 44-64.

[8] M. Kummert, P. Andre, Simulation of a model-based optimal controller for heating systems under realistic hypothesis, in: Proc. of the 9th IBPSA Conference, Montreal, Canada, 2005.

[9] G.P. Henze, D.E. Kalz, S. Liu, C. Felsmann, Experimental analysis of model-based predictive optimal control for active and passive building thermal storage inventory, HVAC\& R Research 11 (2005) 189-213.

[10] R.Z. Freire, G.H. Oliveira, N. Mendes, Predictive controllers for thermal comfort optimization and energy savings, Energy and Buildings 40 (2008) 1353-1365.

[11] M. Castilla, J.D. Alvarez, M. Berenguel, F. Rodríguez, J.L. Guzmán, M. Pérez, A comparison of thermal comfort predictive control strategies, Energy and Buildings 43 (2011) 2737-2746.

[12] P.M. Ferrira, A.E. Ruano, S. Silva, E.Z.E. Conceicao, Neural networks based predictive control for thermal comfort and energy savings in public buildings, Energy and Buildings 55 (2012) 238-251.

[13] F. Oldewurtel, C. N. Jones, M. Morari, A tractable approximation of chance constrained stochastic MPC based on affine disturbance feedback, in: Proc. of 47th IEEE Conference on Decision and Control, Cancun, Mexico, 2008.

[14] F. Oldewurtel, A. Parisio, C. N. Jones, D. Gyalistras, M. Gwerder, V. Stauch, B. Lehmann, M. Morari, Use of model predictive control and weather forecasts for energy efficient building climate control, Energy and Buildings 45 (2012) 15-27. 
[15] Y. Ma, F. Borrelli, Fast stochastic predictive control for building temperature regulation, in: Proc. of American Control Conference, Montreal, Canada, 2012.

[16] X. Zhang, G. Sturzenegger, D. Sturzenegger, M. Morari, Scenario-based MPC for energy-efficient building climate control under weather and occupancy uncertainty, in: Proc. of European Control Conference, Zurich, Switzerland, 2013.

[17] X. Xu, S. Wang, G. Huang, Robust MPC for temperature control of air-conditioning systems concerning on constraints and multitype uncertainties, Building Services Engineering Research and Technology 31 (2010) $39-55$.

[18] M. Maasoumy, A. Sangiovanni-Vincentelli, Optimal control of building hvac systems in the presence of imperfect predictions, in: Proc. of 5th Annual Dynamic Systems and Control Conference joint with the 11th Motion and Vibration Conference, Fort Lauderdale, Florida, 2012.

[19] J. Siroky, F. Oldewurtel, J. Cigler, S. Privara, Experimental analysis of model predictive control for an energy efficient building heating system, Applied Energy 88 (2011) 3079-3087.

[20] S. Atthajariyakul, T. Leephakpreeda, Neural computing thermal comfort index for HVAC systems, Energy Conversion and Management 46 (2005) 2553-2565.

[21] C.C. Federspiel, H. Asada, User-adaptable comfort control for HVAC systems, Journal of dynamic systems, measurement, and control 116 (1994) 474-486.

[22] X. Chen, Q. Wang, J. Srebric, A data-driven state-space model of indoor thermal sensation using occupant feedback for low-energy buildings, Energy and Buildings, 91 (2015) 187-198.

[23] J. Van Hoof, Forty years of Fanger's model of thermal comfort: comfort for all? Indoor Air 18 (2008) 182201.

[24] J. Toftum, Central automatic control or distributed occupant control for better indoor environment quality in the future, Building and environment 45 (2010) 23-28.

[25] S. Barlow, D. Fiala, Occupant comfort in UK offices-How adaptive comfort theories might influence future low energy office refurbishment strategies, Energy and Buildings 39 (2007) 837-846.

[26] Y. Bar-Shalom, X. Li, T. Kirubarajan, Estimation with Application to Tracking and Navigation, John Wiley \& Sons, New York, 2001. 
[27] G. Fraisse, C. Viardot, O. Lafabrie, G. Achard, Development of a simplified and accurate building model based on electrical analogy, Energy and buildings 34 (2002) 1017-1031.

[28] B. Bueno, L. Norford, G. Pigeon, R. Britter, A resistance-capacitance network model for the analysis of the interactions between the energy performance of buildings and the urban climate, Building and Environment 54 (2012) 116-125.

[29] J.D. Bynum, D. E. Claridge, J. M. Curtin, Development and testing of an Automated Building Commissioning Analysis Tool (ABCAT), Energy and Buildings 55 (2012) 607-617.

[30] S. Goyal, P. Barooah, A method for model-reduction of non-linear thermal dynamics of multi-zone buildings, Energy and Buildings 47(2012) 332-340.

[31] DOE, US, Energyplus engineering reference, The Reference to EnergyPlus Calculations, 2014.

[32] A. Prékopa, Stochastic programming, Springer, 1995. 
Fig. 1. Climate chamber layout.

Fig. 2. Single room thermal network model.

Fig. 3. Block diagram for DTS model based MPC.

Fig. 4. Indoor relative humidity used in simulation.

Fig. 5. Clothing insulation level used in simulation.

Fig. 6. Outdoor weather. (a) Top: 3 day outdoor temperature history in March. Bottom: 3 day outdoor temperature history in September. (b) Top: 3 day outdoor relative humidity history in March. Bottom: 3 day outdoor relative humidity history in September.

Fig. 7. Controlled indoor air temperature. (a) Representative Scenario I (March, $E[w]=0$ ). (b) Representative Scenario II (March, $E[w]=-0.2$ ).

Fig.8. Control input. (a) Representative Scenario I (March, $E[w]=0$ ), control input corresponds to heating power.

(b) Representative Scenario II (March, $E[w]=-0.2$ ), control input corresponds to cooling power.

Fig. 9. Actual mean vote (AMV) from virtual occupants. (a) Representative Scenario I (March, $E[w]=0$ ). (b) Representative Scenario II (March, $E[w]=-0.2$ ).

Fig. 10. Time-varying offset parameter of the DTS model estimated by EKF. (a) Representative Scenario I (March, $E[w]=0)$. (b) Representative Scenario II (March, $E[w]=-0.2$ ).

Fig. 11. Pareto frontier of energy consumption versus thermal comfort violation. (a) March, $E[w]=0$; (b) September, $E[w]=0$. 
Table 1. Key parameters of the thermal-comfort chamber experiment.

\begin{tabular}{|c|c|c|c|c|}
\hline $\begin{array}{c}\text { Occupant } \\
\text { clothing } \\
\text { insulation }\end{array}$ & $\begin{array}{c}\text { Occupant } \\
\text { activity } \\
\text { level }\end{array}$ & $\begin{array}{c}\text { Chamber } \\
\text { mean radiant } \\
\text { temperature }\end{array}$ & $\begin{array}{c}\text { Chamber } \\
\text { average } \\
\text { RH }\end{array}$ & $\begin{array}{c}\text { Chamber } \\
\text { average air } \\
\text { velocity }\end{array}$ \\
\hline 0.71 & 1 & $\begin{array}{c}\text { Closely follows } \\
\text { ambient temperature }\end{array}$ & $21 \%$ & $0.135 \mathrm{~m} / \mathrm{s}$ \\
\hline
\end{tabular}

Table 2. Chamber parameters used in the building model.

\begin{tabular}{|c|c|c|}
\hline & Room(air) & Walls \\
\hline Density $\left(\mathrm{Kg} / \mathrm{m}^{3}\right)$ & 1.16 & 2000 \\
\hline Specific heat Cp (J/Kg-K) & 1007 & 935 \\
\hline Conduction coefficient k (W/m-K) & & 0.744 \\
\hline Convection coefficient h (W/m²-K) & & 5 \\
\hline Length (m) & 6 & \\
\hline Width (m) & 6 & \\
\hline Height (m) & 3 & \\
\hline Air mass flow rate (kg/s) & 0.139 & \\
\hline Thickness (m) & & 0.15 \\
\hline
\end{tabular}


Table 3. Performance statistics (average of 20 simulation runs).

\begin{tabular}{|c|c|c|c|c|c|c|}
\hline $\begin{array}{l}\text { Case } \\
\text { Number }\end{array}$ & $\begin{array}{l}\text { Weather } \\
\text { Condition }\end{array}$ & $\begin{array}{l}\text { Expected Value } \\
\text { of Noise in } \\
\text { Virtual Occupant }\end{array}$ & Controller & Energy(E): J & $\begin{array}{c}\text { Violation } \\
\text { Ratio(VR): \% }\end{array}$ & $\begin{array}{c}\text { Integration of } \\
\text { Violation(IOV) }\end{array}$ \\
\hline 1 & \multirow{12}{*}{ March } & \multirow{4}{*}{$E[w]=0$} & RuleBased-PI & $2.38 \mathrm{e} 8$ & 37.10 & 13.40 \\
\hline 2 & & & MPC-PMV & $2.07 \mathrm{e} 8$ & 49.39 & 8.99 \\
\hline 3 & & & CEMPC-DTS & $2.19 \mathrm{e} 8$ & 51.84 & 12.17 \\
\hline 4 & & & CCMPC-DTS & $2.38 \mathrm{e} 8$ & 15.71 & 2.66 \\
\hline 5 & & \multirow{4}{*}{$E[w]=0.2$} & RuleBased-PI & $2.38 \mathrm{e} 8$ & 7.53 & 1.96 \\
\hline 6 & & & MPC-PMV & $2.07 \mathrm{e} 8$ & 0.62 & 0.054 \\
\hline 7 & & & CEMPC-DTS & $1.84 \mathrm{e} 8$ & 49.74 & 10.65 \\
\hline 8 & & & CCMPC-DTS & $2.04 \mathrm{e} 8$ & 12.12 & 1.65 \\
\hline 9 & & \multirow{4}{*}{$E[w]=-0.2$} & RuleBased-PI & $2.38 \mathrm{e} 8$ & 61.09 & 43.00 \\
\hline 10 & & & MPC-PMV & $2.07 \mathrm{e} 8$ & 99.08 & 57.37 \\
\hline 11 & & & CEMPC-DTS & $2.56 \mathrm{e} 8$ & 51.93 & 14.53 \\
\hline 12 & & & CCMPC-DTS & $2.74 \mathrm{e} 8$ & 18.35 & 4.25 \\
\hline 13 & \multirow{12}{*}{ September } & \multirow{4}{*}{$E[w]=0$} & RuleBased-PI & $1.96 \mathrm{e} 8$ & 41.16 & 22.82 \\
\hline 14 & & & MPC-PMV & $1.82 \mathrm{e} 8$ & 49.27 & 9.12 \\
\hline 15 & & & CEMPC-DTS & $1.70 \mathrm{e} 8$ & 49.39 & 13.81 \\
\hline 16 & & & CCMPC-DTS & $1.86 \mathrm{e} 8$ & 19.44 & 3.60 \\
\hline 17 & & \multirow{4}{*}{$E[w]=0.2$} & RuleBased-PI & $1.96 \mathrm{e} 8$ & 71.48 & 55.32 \\
\hline 18 & & & MPC-PMV & $1.82 \mathrm{e} 8$ & 99.13 & 57.37 \\
\hline 19 & & & CEMPC-DTS & $2.03 \mathrm{e} 8$ & 53.65 & 14.43 \\
\hline 20 & & & CCMPC-DTS & $2.25 \mathrm{e} 8$ & 20.23 & 3.62 \\
\hline 21 & & \multirow{4}{*}{$E[w]=-0.2$} & RuleBased-PI & $1.96 \mathrm{e} 8$ & 15.78 & 6.91 \\
\hline 22 & & & MPC-PMV & $1.82 \mathrm{e} 8$ & 0.52 & 0.0291 \\
\hline 23 & & & CEMPC-DTS & $1.37 \mathrm{e} 8$ & 42.05 & 11.17 \\
\hline 24 & & & CCMPC-DTS & $1.53 \mathrm{e} 8$ & 17.43 & 3.26 \\
\hline
\end{tabular}




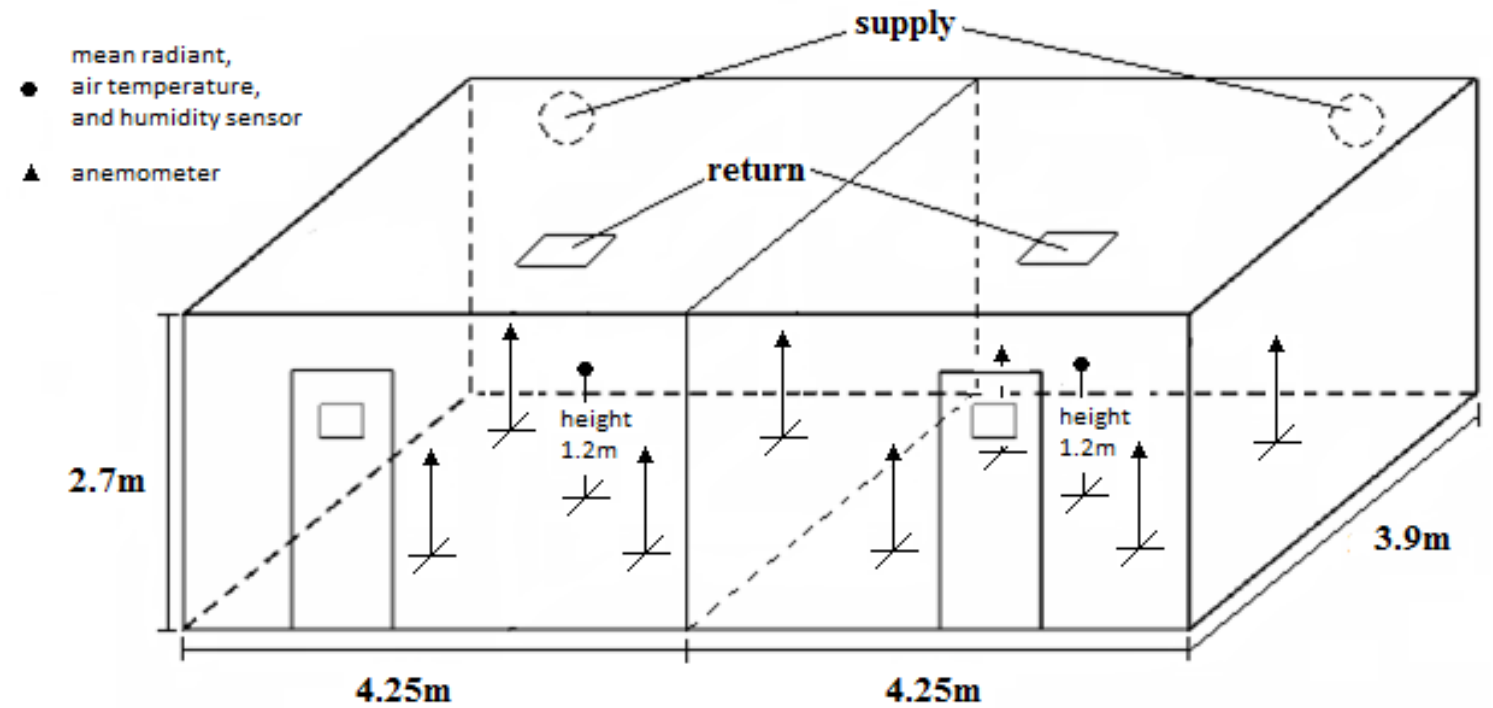

Fig. 1. Climate chamber layout.

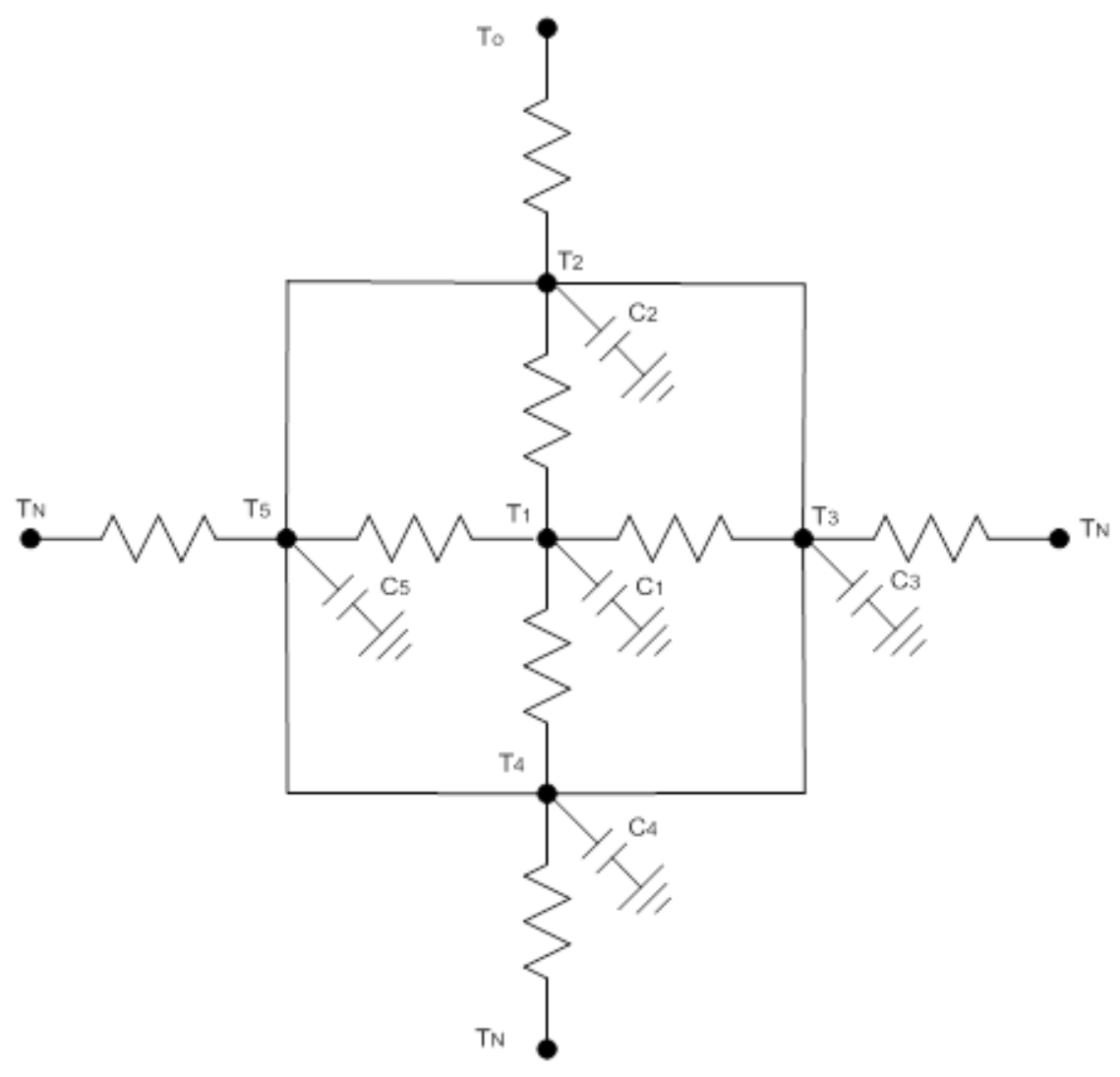

Fig. 2. Single room thermal network model. 


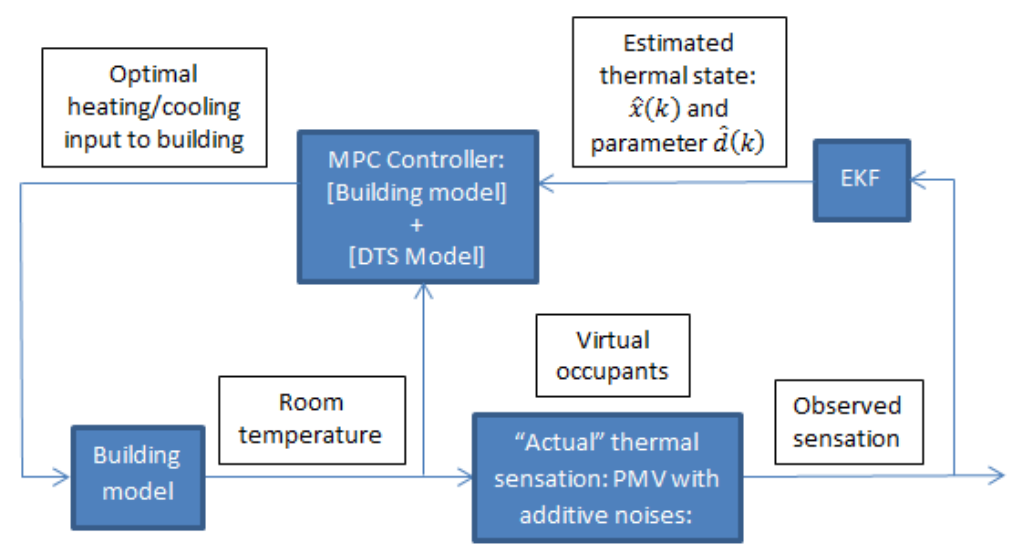

Fig.3. Block diagram for DTS model based MPC.

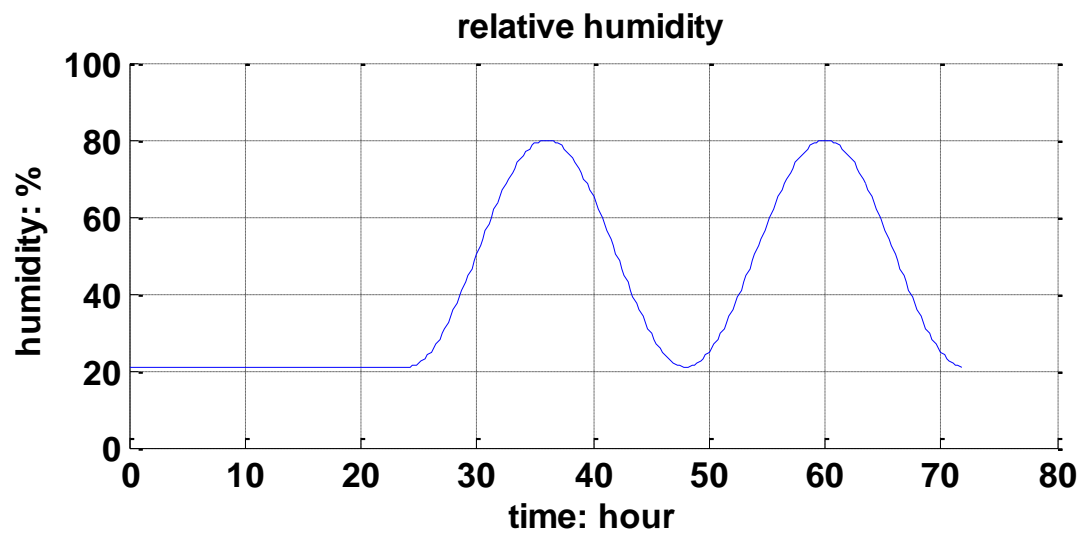

Fig. 4. Indoor relative humidity used in simulation. 


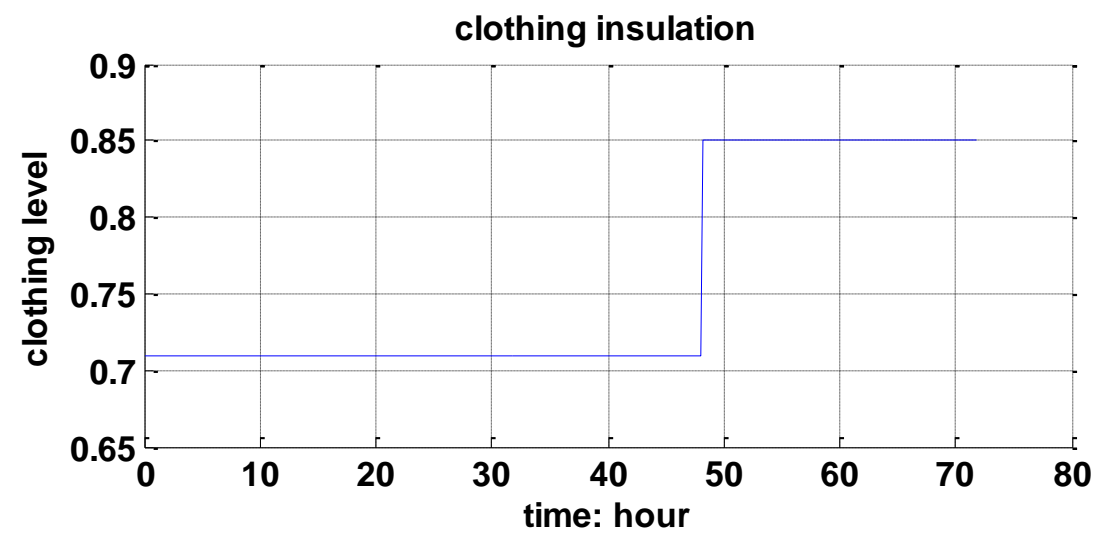

Fig. 5. Clothing insulation level used in simulation. 

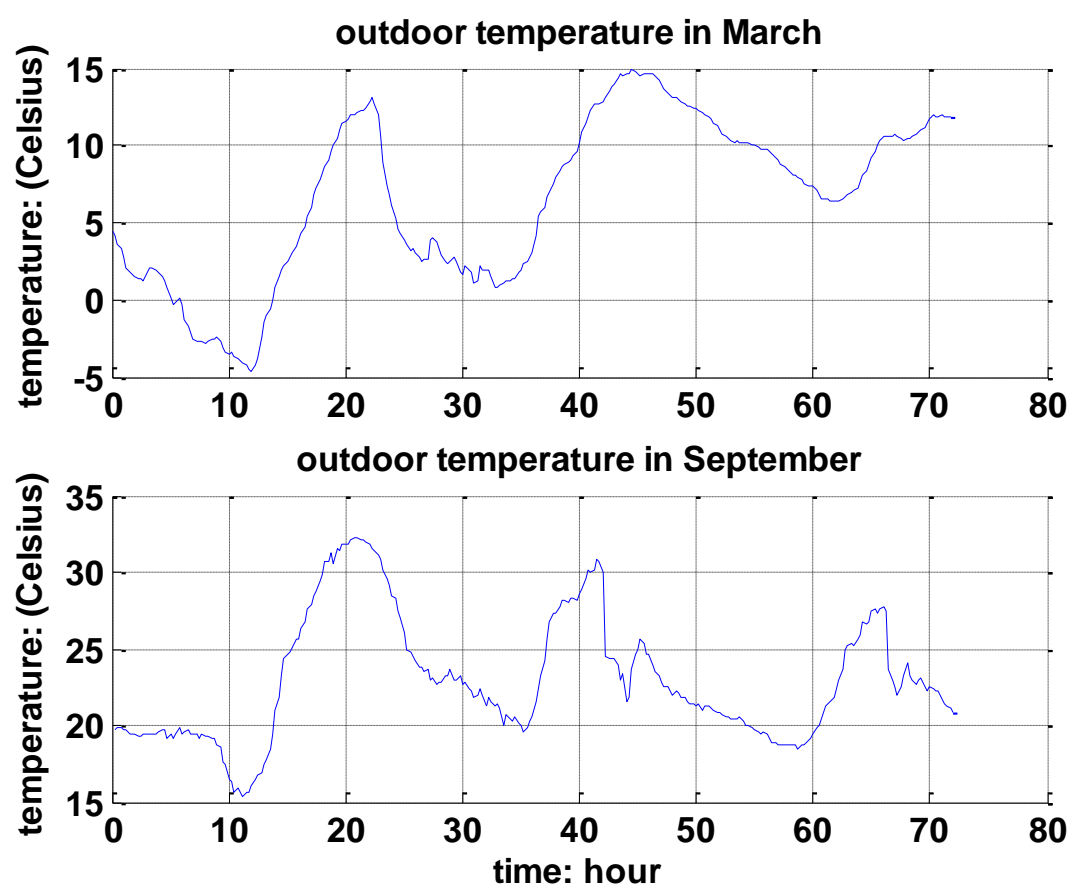

(a)
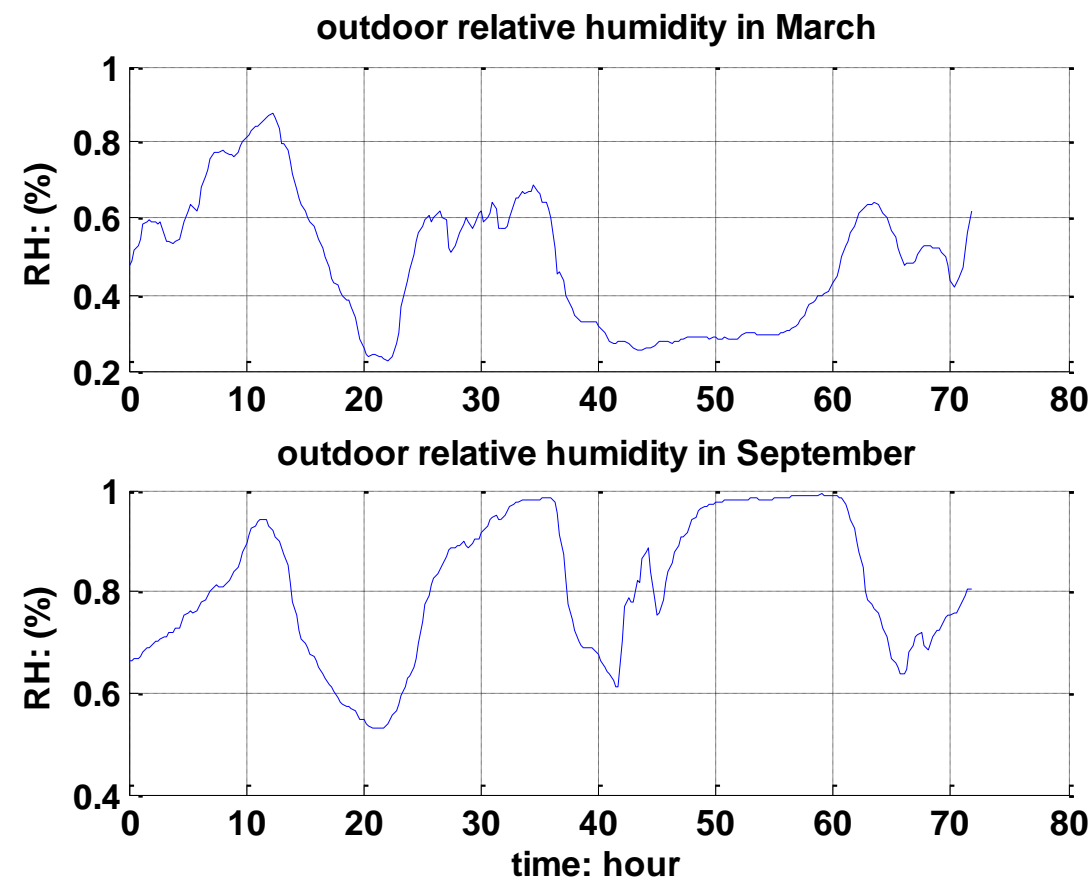

(b)

Fig. 6. Outdoor weather. (a) Top: 3 day outdoor temperature history in March. Bottom: 3 day outdoor temperature history in September. (b) Top: 3 day outdoor relative humidity history in March. Bottom: 3 day outdoor relative humidity history in September. 


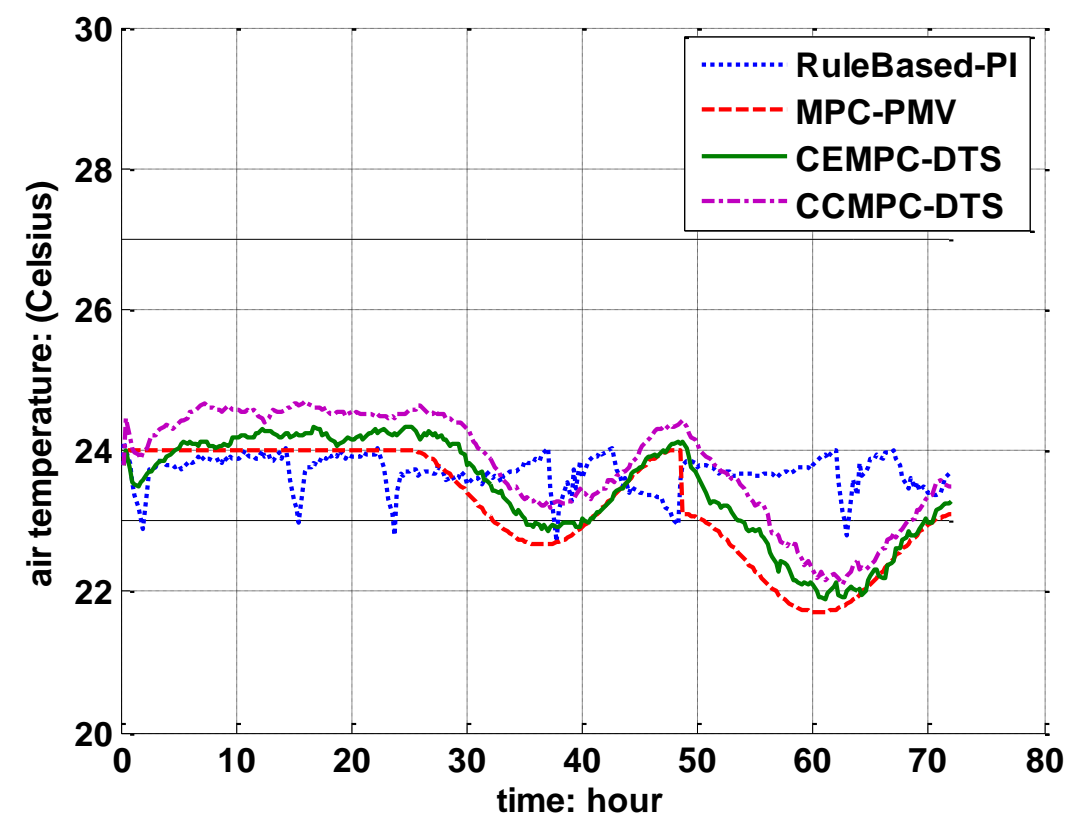

(a)

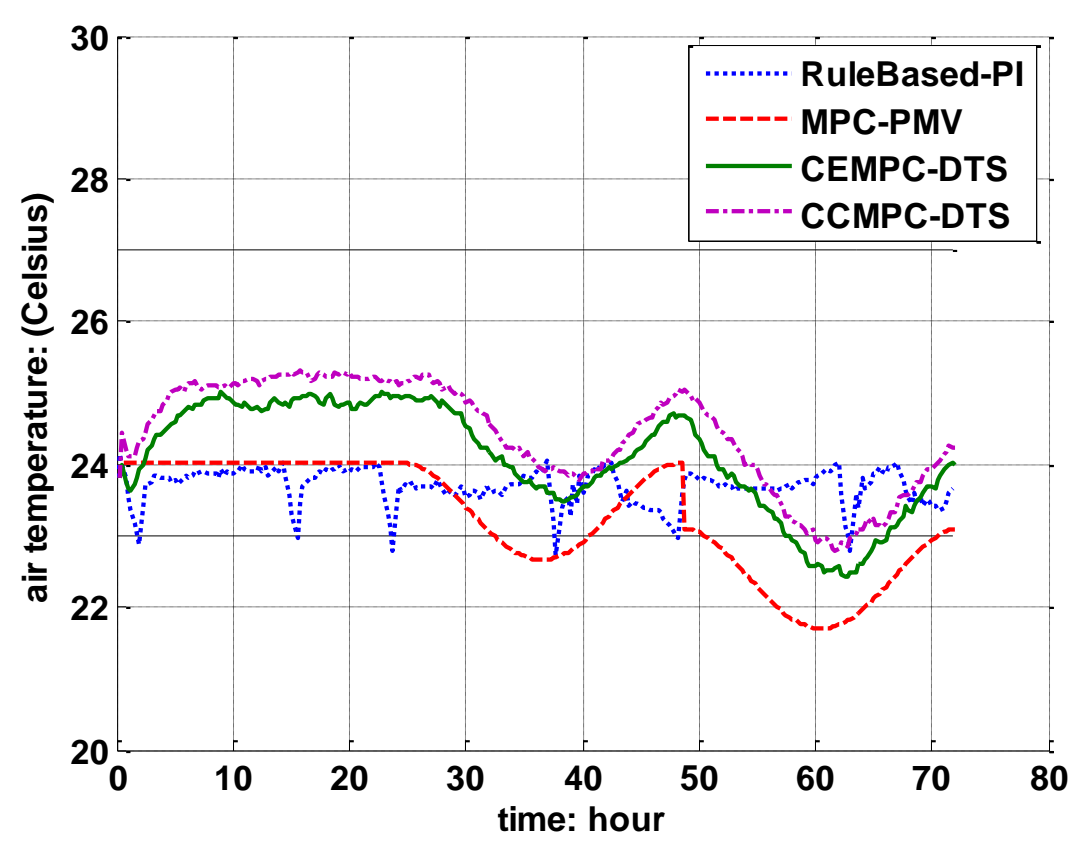

(b)

Fig. 7. Controlled indoor air temperature. (a) Representative Scenario I (March, $E[w]=0$ ). (b) Representative Scenario II (March, $E[w]=-0.2$ ). 


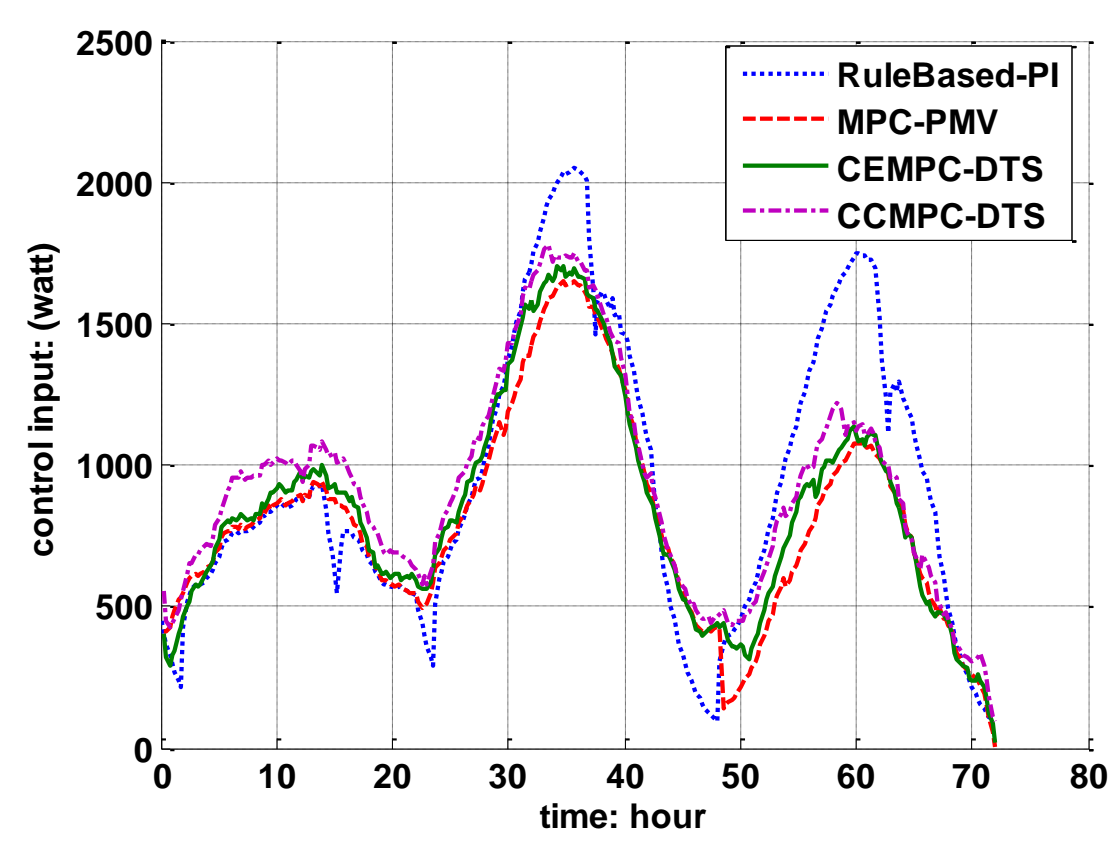

(a)

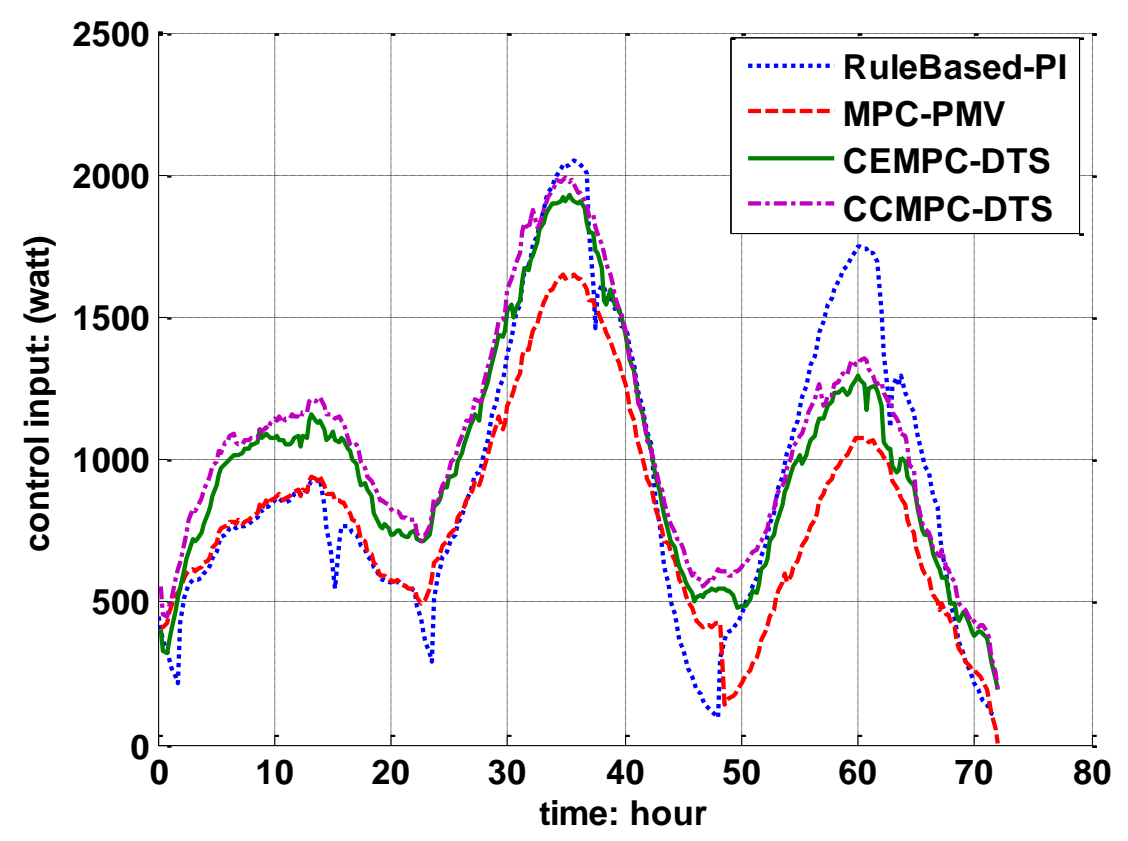

(b)

Fig.8. Control input. (a) Representative Scenario I (March, $E[w]=0$ ), control input corresponds to heating power. (b) Representative Scenario II (March, $E[w]=-0.2$ ), control input corresponds to cooling power. 

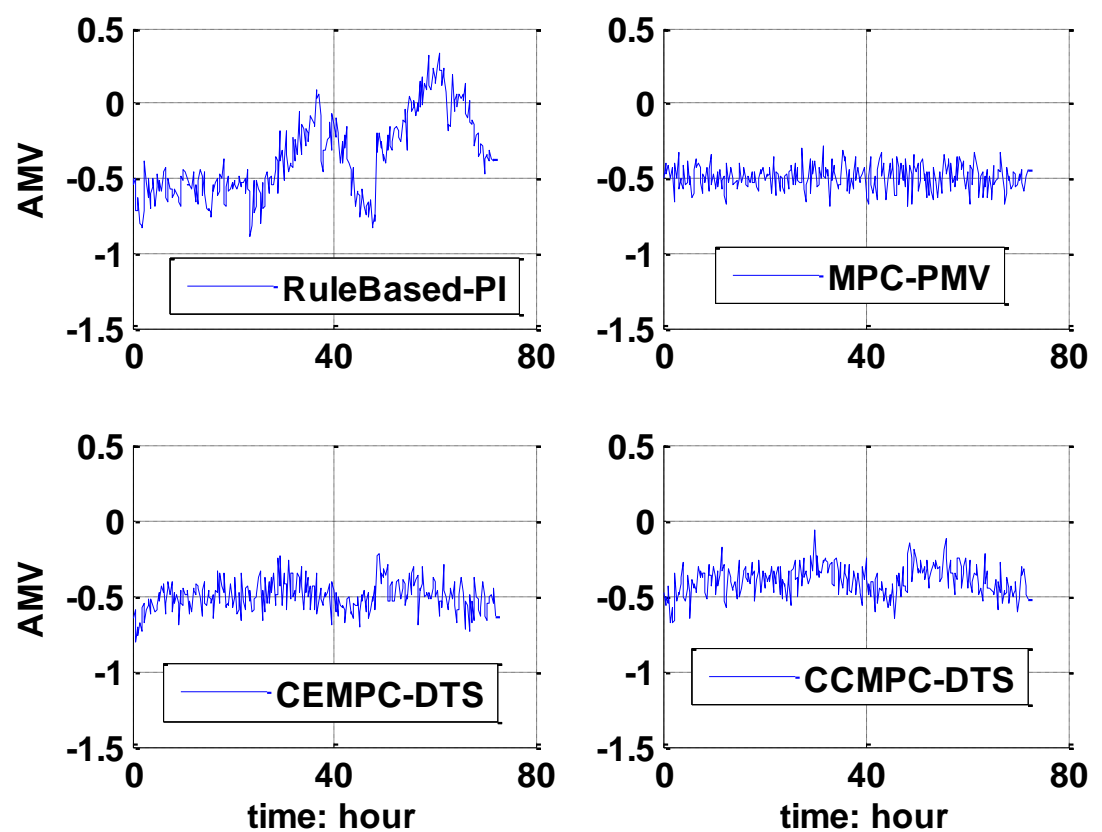

(a)
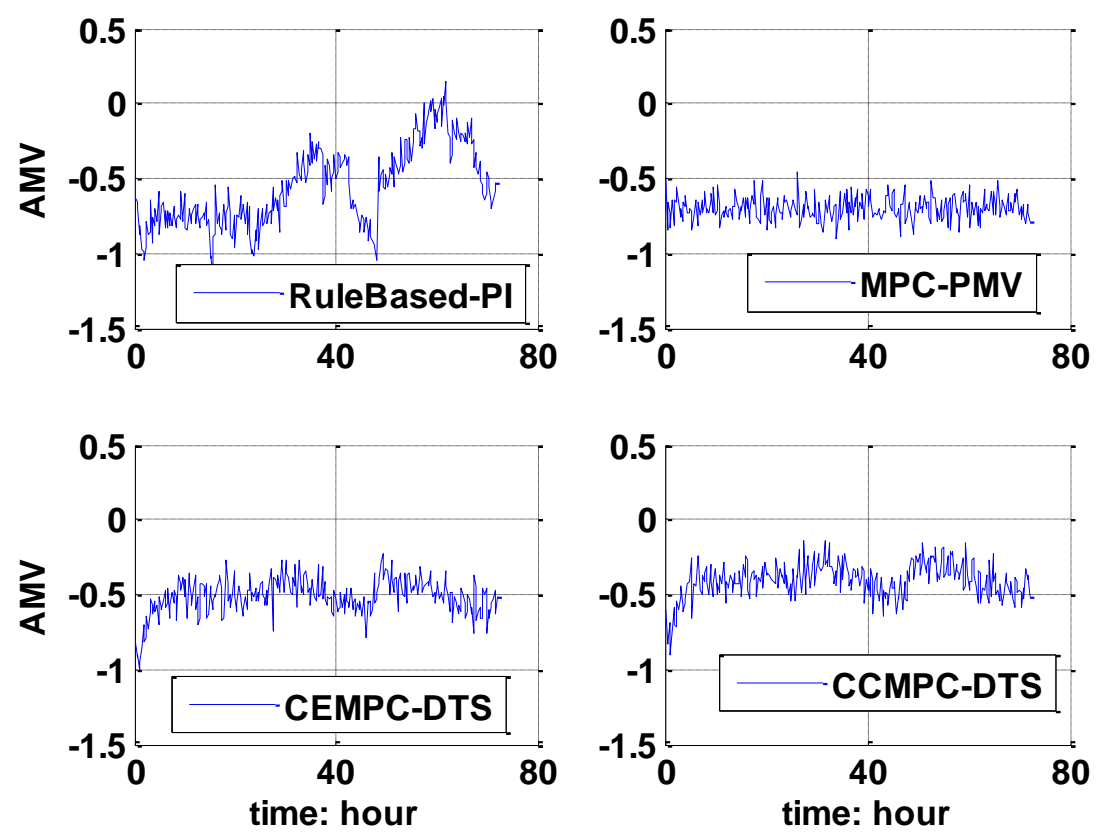

(b)

Fig. 9. Actual mean vote (AMV) from virtual occupants. (a) Representative Scenario I (March, $E[w]=0)$. (b) Representative Scenario II (March, $E[w]=-0.2$ ). 


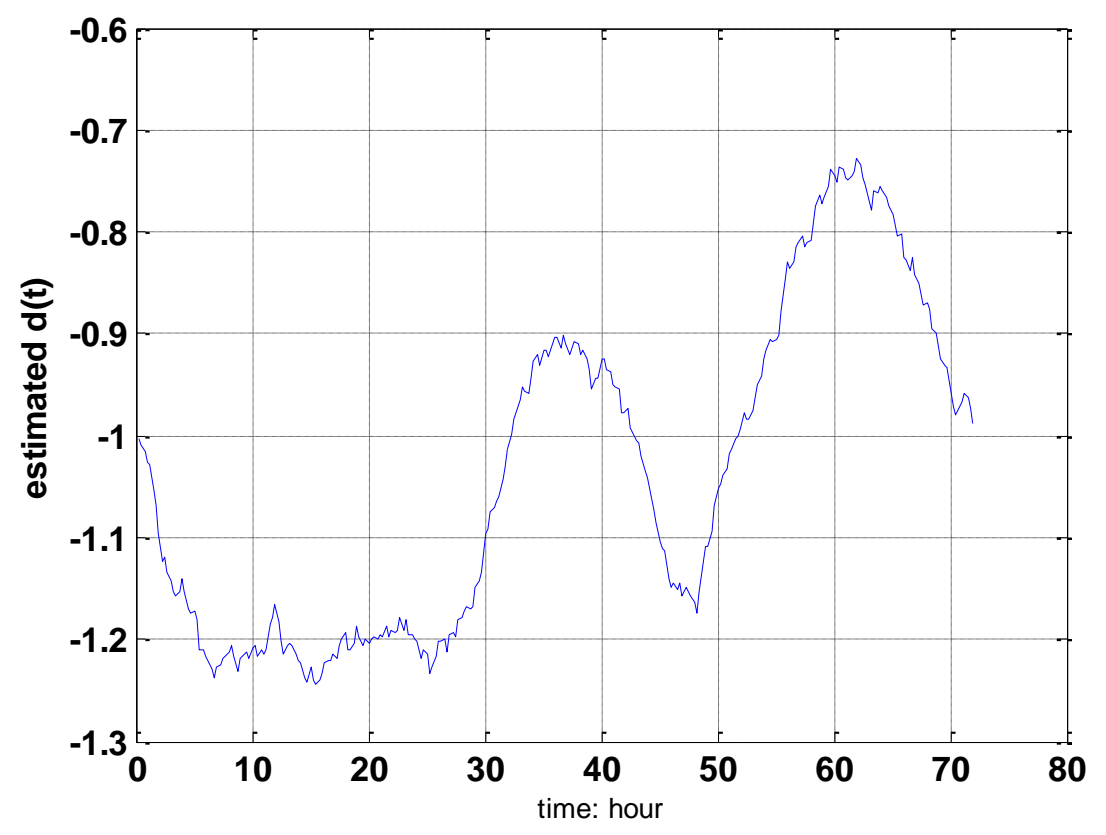

(a)

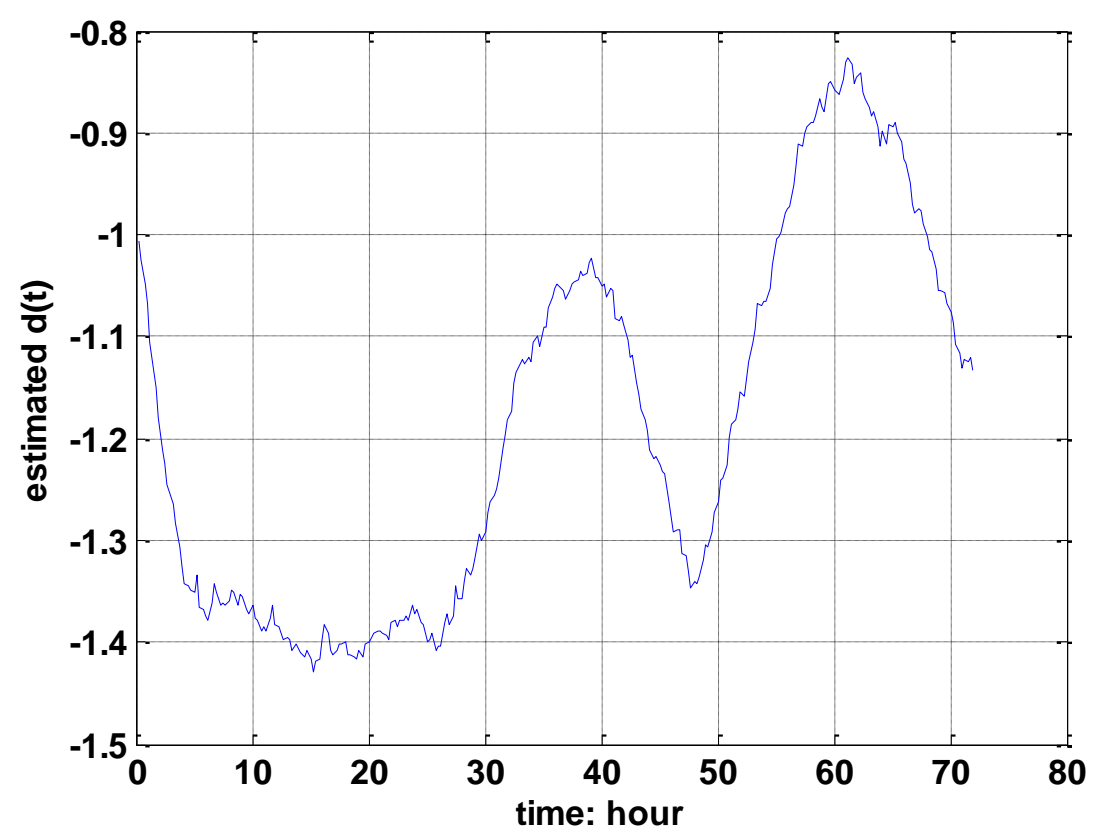

(b)

Fig. 10. Time-varying offset parameter of the DTS model estimated by EKF. (a) Representative Scenario I (March, $E[w]=0$ ). (b) Representative Scenario II (March, $E[w]=-0.2$ ). 


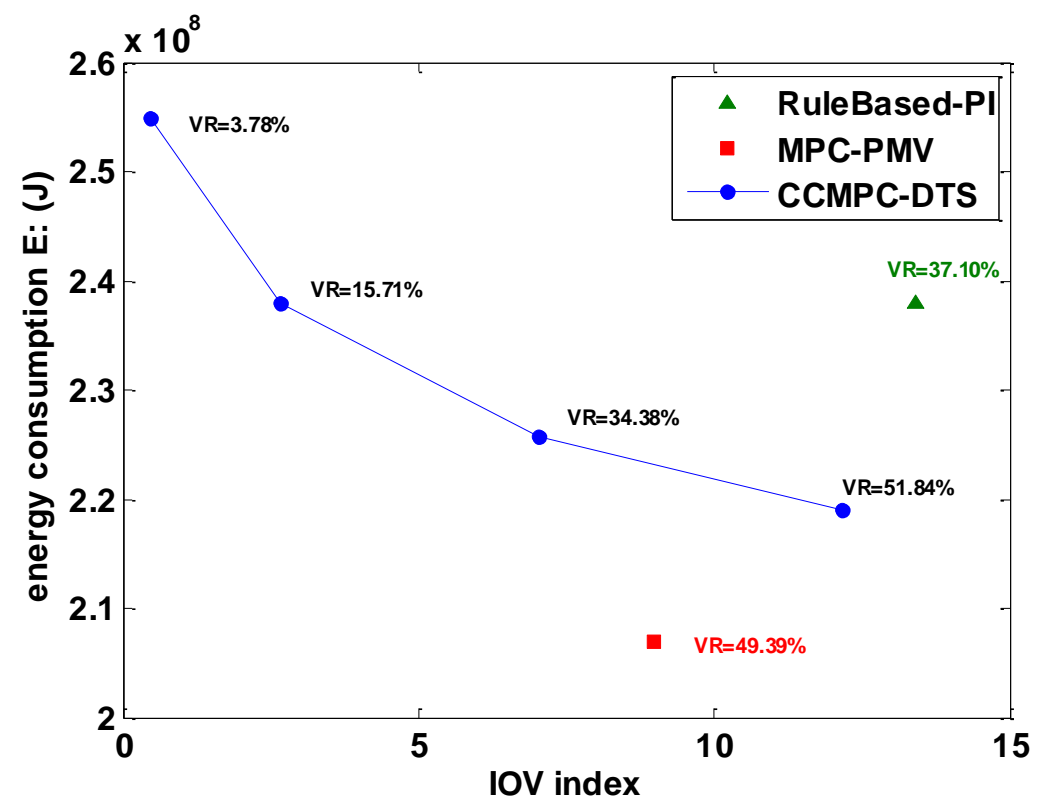

(a)

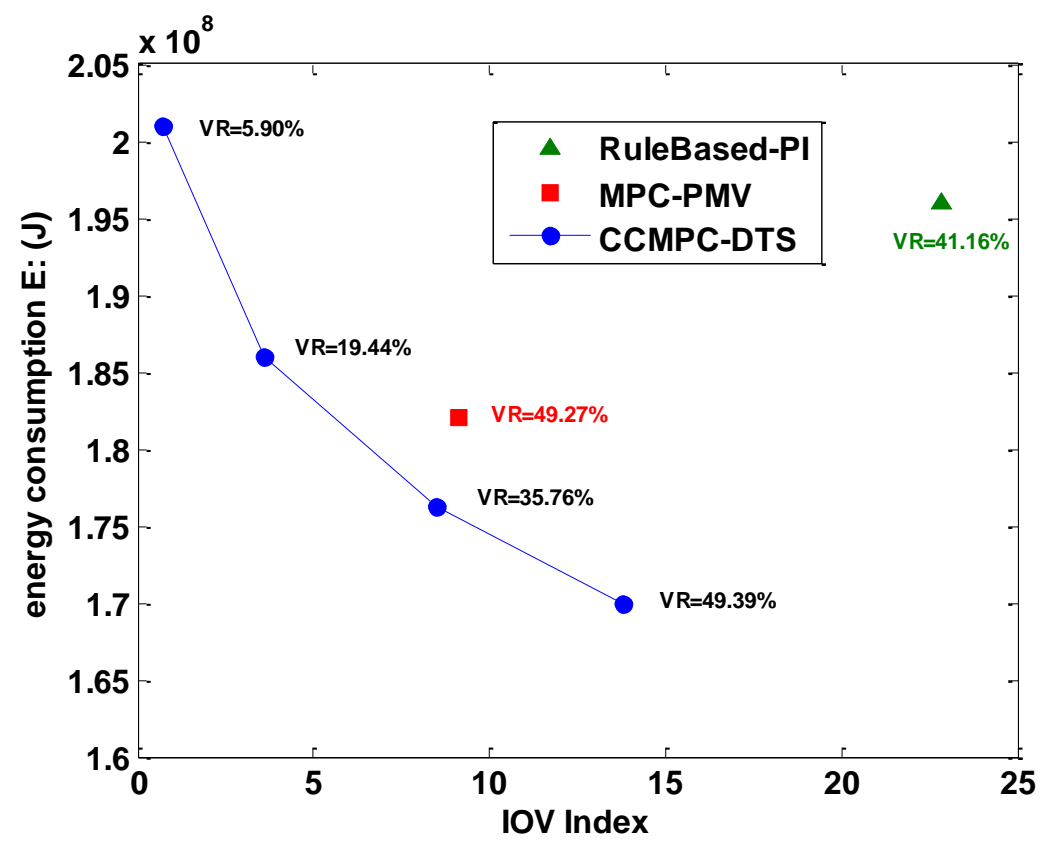

(b)

Fig. 11. Pareto frontier of energy consumption versus thermal comfort violation. (a) March, $E[w]=0$; (b) September, $E[w]=0$. 